\title{
HgSe BASED MIXED CRYSTALS DOPED WITH Fe RESONANT DONORS
}

\author{
W. DOBROWOLSKI \\ Institute of Physics, Polish Academy of Sciences \\ Al. Lotników 32/46, 02-668 Warszawa, Poland
}

(Received October 9, 1995)

\begin{abstract}
The article reviews the physical properties of semimagnetic semiconductors of the type $\mathrm{Hg}_{1-x-y} \mathrm{Fe}_{x} \mathrm{~A}_{y}^{\mathrm{II}} \mathrm{Se}_{1-z} \mathrm{~B}_{z}^{\mathrm{VI}}$ and $\mathrm{Hg}_{1-x-y} \mathrm{Fe}_{x} \mathrm{Mn}_{y}$ Se. Optical, magnetooptical, transport and magnetotransport experiments showed that in $\mathrm{Hg}_{1-x} \mathrm{Fe}_{x}$ Se substitutional iron forms a resonant donor state whose energy is superimposed on the conduction band continuum. Resulting anomalous properties of electron scattering rate, i.e. strong enhancement of electron mobility (or drop of Dingle temperature), which occur in $\mathrm{Hg}_{1-x} \mathrm{Fe}_{x} \mathrm{Se}$ at low temperatures in a certain Fe concentration range, are described. Next, theoretical models describing this anomalous reduction of the scattering rate are discussed. The description of thermomagnetic, optical, magnetooptical and magnetic properties of $\mathrm{Hg}_{1-x} \mathrm{Fe}_{x} \mathrm{Se}$, with emphasis on features originating from the peculiar iron level position in the band structure of $\mathrm{Hg}_{1-x} \mathrm{Fe}_{x} \mathrm{Se}$, conclude the first part of the present paper. In the second part the physical properties of the semiconducting alloys $\mathrm{Hg}_{1-x} \mathrm{Mn}_{x} \mathrm{Se}: \mathrm{Fe}, \mathrm{Hg}_{1-v} \mathrm{Cd}$ Se:Fe, $\mathrm{Hg}_{1-x} \mathrm{Zn}_{x} \mathrm{Se}: \mathrm{Fe}, \mathrm{HgSe}_{1-x} \mathrm{Te}_{x}: \mathrm{Fe}$ and $\mathrm{HgSe}_{1-x} \mathrm{~S}_{x}: \mathrm{Fe}$ are described. In particular, the dependence of the position of the Fe resonant donor state in the band structure on the crystal composition is discussed. The values of predicted $\Gamma_{6}$ and $\Gamma_{8}$ band offsets between $\mathrm{HgSe}$ and $\mathrm{CdSe}, \mathrm{HgTe}, \mathrm{MnSe}$ and $\mathrm{ZnSe}$ are given. The considerable attention is paid to the discussion of the mechanism limiting the electron mobility in the mixed alloys. Finally, topics which have not been explicitly covered in this review are mentioned and open problems are discussed.
\end{abstract}

PACS numbers: 72.20 .Fr, 72.10.Fk, 71.55.Gs

\section{Introduction}

HgSe crystallizes in the zinc blende structure. The zero energy gap is situated in the $\Gamma$ point of the Brillouin zone. The symmetry of the conduction and heavy hole bands at the center of the Brillouin zone corresponds to $\Gamma_{8}$ representation whereas that of the light hole band to $\Gamma_{6}$ (Gałązka et al. [1] and Seiler et al. [2]). 
Such sequence of the bands, reversed to the order occurring in wide-gap II-VI materials, like CdTe, is called "inverted" band ordering. It was proposed for the first time by Groves and Paul [3] to explain the properties of $\alpha$-Sn. The parameters describing the band structure of $\mathrm{HgSe}$ in the vicinity of the $\Gamma$ point of the Brillouin zone are gathered in Table. Although these parameters obtained by two methods differ, when applied to calculations they result in very close values of conduction band effective masses, Fermi energy etc.

TABLE

The band parameter of HgSe at $4.2 \mathrm{~K}$ from Shubnikov-de Haas effect (Galązka et al. [1]) and interband magnetooptics studies (Dobrowolska et al. [88]).

\begin{tabular}{l|c|c|c|c}
\hline \hline \multicolumn{1}{c|}{ Material parameter } & Symbol & SDH effect data & Magnetooptics data & Units \\
\hline Energy gap $E_{\Gamma_{6}}-E_{\Gamma_{8}}$ & $E_{0}$ & -0.22 & -0.274 & $\mathrm{eV}$ \\
Kane's momentum & & & & \\
$\quad$ matrix element & $P$ & $7.2 \times 10^{-8}$ & $7.1 \times 10^{-8}$ & $\mathrm{eV} \mathrm{cm}$ \\
Spin-orbit splitting & $\Delta$ & 0.45 & 0.39 & $\mathrm{eV}$ \\
Higher band parameters & $\gamma_{1}$ & 3 & 0.1 & \\
& $\gamma_{2}$ & -0.5 & - & \\
& $\gamma_{3}$ & 0.17 & - & \\
& $\gamma$ & - & -0.5 & \\
& $\kappa$ & -0.9 & -1 &
\end{tabular}

HgSe easily forms solid solutions with many semiconducting compounds. It constitutes crystals of arbitrary composition with other symmetry-induced zero gap II-VI semiconductors, i.e. HgTe and HgS. Alloying with wide gap II-VI semiconductors like $\mathrm{ZnSe}$ or CdSe results in a material with an energy gap determined by content (mole fraction $x$ ) of $\mathrm{Zn}$ or $\mathrm{Cd}$. In such alloys with the increasing quantity of $\mathrm{Zn}$ or $\mathrm{Cd}$ the light hole band moves upwards and at some specific $x$ coincides with the position of the conduction band and heavy hole band. In materials with greater $x$, above this point the conduction band belongs to $\Gamma_{6}$ representation, while light and heavy holes band are of $\Gamma_{8}$ representation. Similar behavior of the energy gap and bands ordering can be observed in alloys of $\mathrm{HgSe}$ with the magnetic semiconductor $\mathrm{MnSe}$. The semimagnetic semiconductors obtained in this case, in addition to the properties characteristic of small gap semiconductors, if we consider a small $x$ limit, show a very strong sensitivity of the band edge energies to the temperature and magnetic fields (for recent reviews see e.g. Kossut and Dobrowolski [4] or Dietl [5]). This sensitivity has its origin in an exchange coupling of the conduction (or valence band) electrons to magnetic moments localized on the magnetic component of the compound.

Iron, which in the $\mathrm{HgSe}$ lattice substitutes for $\mathrm{Hg}$ in the cation position, was found to be a donor whose ground state energy is superimposed on the conduction band continuum. In other words, $\mathrm{Fe}$ ions in $\mathrm{HgSe}$ are resonant donors. This peculiar location of the Fe energy state within the conduction band is a source of many anomalies observed in electron transport, optical and magnetic properties, making the material very attractive for investigation. 
The solubility of $\mathrm{Fe}$ in $\mathrm{HgSe}$ is rather high. Crystals of solid solution of $\mathrm{Fe}$ in $\mathrm{HgSe}, \mathrm{Hg}_{1-x} \mathrm{Fe}_{x} \mathrm{Se}$, may be grown in zinc-blende structure with molar fraction of Fe up to $x \approx 0.2$ (Mycielski [6]). With increasing amount of Fe the energy distance between $\Gamma_{8}$ conduction and $\Gamma_{6}$ light hole band decreases to zero, and the semimetal-semiconductor transition point according to the most recent evaluations is reached at $x=0.06$ (Komirenko et al. [7]).

\section{HgSe:Fe \\ 2.1. The location of $\mathrm{Fe}^{2+}$ state in the band structure of $\mathrm{HgSe}$}

The $\mathrm{Fe}^{2+}$ in II-VI compounds is usually a donor giving energy level in the forbidden gap (see e.g. Langer and Heinrich [8]). A different situation was stated in IIgSe crystal. Various experiments showed that the $\mathrm{Fe}^{2+}$ state in this compound is degenerate with the conduction band and is located approximately $200 \mathrm{meV}$ above the bottom of the conduction band at $4.2 \mathrm{~K}$ (Mycielski et al. [9], Pool et al. [10], Dobrowolski et al. [11], Reich et al. [12], Kowalski et al. [13], Szuszkiewicz et al. [14]). On account of its energetic position $\mathrm{Fe}$ in $\mathrm{HgSe}$ acts as a resonant donor and it is subject to a self-ionization process $\mathrm{Fe}^{2+} \rightarrow \mathrm{Fe}^{3+}+e_{c . b .}$. provided that there are available unoccupied states in the conduction band below the energy corresponding to the $\mathrm{Fe}^{2+}$ state. Under this condition, at low temperatures, the number of conduction electrons is roughly equal to the number of Fe impurities present in the crystal. At the electron concentration $n_{\mathrm{e}} \approx 4.5 \times 10^{18} \mathrm{~cm}^{-3}$, the Fermi energy reaches the $\mathrm{Fe}^{2+}$ level and the further self-ionization becomes impossible. Even stronger doping with Fe results in a part of iron ions left in the $\mathrm{Fe}^{2+}$ state. At such high doping levels, coexisting $\mathrm{Fe}^{3+}$ and $\mathrm{Fe}^{2+}$ ions are said to create a mixed valence system. A direct evidence for this behavior is provided by the dependence of a concentration of free electrons on the iron concentration (see Fig. 1). At low doping levels $n_{\mathrm{e}}$ is proportional to a number of $\mathrm{Fe}$ ions present in the crystal, and above a certain level of doping $\left(n_{\mathrm{Fe}}^{*} \approx 4.5 \times 10^{18} \mathrm{~cm}^{-3}\right)$, when the Fermi level becomes pinned to the $\mathrm{Fe}$ level, the electron concentration remains constant.

The study of the Fe donor energy as a function of the temperature and the crystal composition was performed by Pool et al. [10]. By fitting changes of the electron concentration, induced by the temperature, in $\mathrm{Hg}_{1-x} \mathrm{Fe}_{x}$ Se samples for $x \leq 0.12$, it was found that a correct description may be achieved only if there exists a possibility of a relative shift of the $\mathrm{Fe}$ level $E_{\mathrm{Fe}}$ in respect to band edges. The authors found that this dependence may be expressed by equation

$$
E_{\mathrm{Fe}}(T)=E_{\mathrm{Fe}}(0)+\alpha\left|E_{0}(0)-E_{0}(T)\right|[\mathrm{eV}],
$$

where $E_{\mathrm{Fe}}(x)=0.21+0.52 x \mathrm{eV}$ describes the dependence of the $E_{\mathrm{Fe}}$ on molar fraction $x$ and $E_{0}$ is $\Gamma_{6}-\Gamma_{8}$ energy gap. An adjustable parameter $\alpha$ varies from 0.24 to 0.16 for values of $x$ in the range from 0.0003 to 0.01 .

Another demonstration of the degeneracy of the Fe level with the conduction band was found in the experiments performed in high magnetic fields. The magnetoreflection experiment (Dobrowolski et al. [11]) provided clear evidence that when $n_{\mathrm{Fe}} \geq n_{\mathrm{Fe}}^{*}$ the Fermi level is pinned to the $\mathrm{Fe}^{2+}$ state. In a quantizing magnetic field, due to this pinning, the oscillations of the free carrier concentration, in a place 


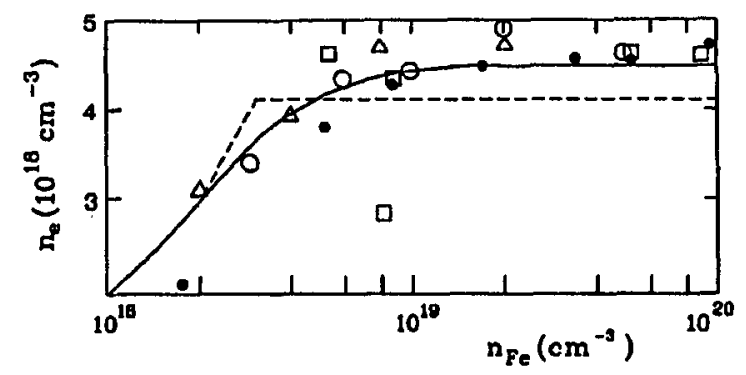

Fig. 1. Electron concentration in the conduction band in $\mathrm{HgSe}: \mathrm{Fe}$ as a function of Fe concentration. Experimental points at $4 \mathrm{~K}$ are taken from: full circles [10], open circles [11], squares [87], triangles [27]. The broken line was calculated with $E_{\mathrm{Fe}}=210 \mathrm{meV}$ without the inter-donor Coulomb interaction, while the continuous curve represents the calculation including that interaction within the short-range correlation model [34] (Courtesy of Z. Wilamowski).

of otherwise present small oscillations of the Fermi level, should be observed. The free carrier concentration is directly related to the plasma frequency $\left(\omega_{\mathrm{p}} \propto \sqrt{n_{\mathrm{e}}}\right)$. In the parallel Voigt configuration $(E \| H)$ the plasma edge does not depend on a magnetic field and it is determined solely by a plasma frequency. Oscillations of the plasma frequency should in turn lead to an oscillation of reflectivity as a function of a magnetic field. The experiment confirmed the expected phenomenon, the oscillations periodic in $1 / B$ and with a relative amplitude of about $1 \%$ at $7 \mathrm{~T}$ were detected.

Independent confirmations of the quoted model, predicting oscillations of the electron concentration, were drawn from the observation of oscillations of the Hall coefficient in a magnetic field (Dobrowolski et al. [11] and von Ortenberg et al. [15]).

The dependence of the amplitude of electron concentration oscillations as a function of a magnetic field was theoretically analyzed by Kossut and Dobrowolski [16]. The authors assumed the rectangular density-of-state of resonant donors with the width $W$ and the height equal to $N_{\mathrm{R}} / W$, where $N_{\mathrm{R}}$ is the donor concentration. The concentration of both conduction electrons and occupied donors was calculated assuming the strong degeneracy of the electron gas. By performing the Poisson transformation and retaining only the first term in the obtained harmonic series, the following form of the neutrality equation was obtained:

$$
\frac{2 \exp (-2 \pi \Gamma / \hbar \omega)}{(2 \pi L)^{3} N_{\mathbf{R}}} \cos \left(\frac{2 \pi E_{\mathrm{F}}}{\hbar \omega}-\frac{5}{4} \pi\right)=\frac{1}{2}-\frac{1}{W}\left(E_{\mathrm{F}}-E_{\mathrm{R}}\right),
$$

where $L=(\hbar c / e B)^{1 / 2}$ is the magnetic length, $\hbar \omega$ is the cyclotron energy, $E_{\mathrm{R}}$ is the distance of the resonant state energy center from the conduction band bottom, and $E_{\mathrm{F}}$ is the Fermi energy that is to be found by solving the above equation. $\Gamma$ is the broadening of the conduction band Landau levels.

The dimensionless parameter $P=(W / R)\left[(2 \pi L)^{3} N_{\mathrm{R}}\right]^{1 / 2} \exp (-2 \pi \Gamma / \hbar \omega)$ determines the behavior of the Fermi energy. If $P \ll 1$, which is the case of $\mathrm{HgSe}: \mathrm{Fe}^{*}$,

\footnotetext{
${ }^{*} P=3.8 \times 10^{-6} W$, where $W$ is expressed in meV, for $\mathrm{HgSe}: \mathrm{Fe}$ in the magnetic field $B=10 \mathrm{~T}$.
} 
the resulting $E_{\mathrm{F}}$ is almost independent of the magnetic field, and consequently, the conduction electron concentration $n(B)$ oscillates. However, the amplitude of these oscillations does not depend sensitively on the width of the resonant state. Therefore it was concluded that it is practically impossible to determine the resonant state width basing on the amplitude of $n(B)$ oscillations.

The presence of $\mathrm{Fe}$ in the $\mathrm{Fe}^{3+}$ state was documented directly by EPR studies (Dobrowolski et al. [11] and Wilamowski et al. [17]). The symmetry of EPR lines confirms that $\mathrm{Fe}$ occupies the cation site. The lifetime of $\mathrm{Fe}^{3+}-$ as judged by an extremely small width of EPR lines - was found to be very long $\left(10^{-9} \mathrm{~s}\right)$ indicating that the hybridization of the iron state and the conduction band is negligible. Also Mössbauer spectroscopy studies of $\mathrm{Hg}_{1-x} \mathrm{Fe}_{x}$ Se provided straight evidence of the fact that $\mathrm{Fe}$ is present in this compound in two charge states, $\mathrm{Fe}^{2+}$ and $\mathrm{Fe}^{3+}$ (Nowik et al. [18]).

The measurements of the plasma edge splitting in the magnetic field in the Faraday geometry yielded the effective mass of the electrons at the Fermi level (Mycielski et al. [19]). The derived value of the effective mass occurred to be, within an experimental accuracy, in good agreement with those derived from Shubnikov-de Haas (Dobrowolski et al. [11], Vaziri and Reifenberger [20]) and de Haas-van Alphen (Miller and Reifenberger [21, 22]) oscillations as well as from magnetoabsorption (Portugall et al. [23]) and strip-line (Buchholtz-Stepputtis et al. $[24,25])$ spectra.

The values of the effective mass found in these investigations reproduce the values typical of $\mathrm{HgSe}$ with the comparable carrier concentration produced by other nonresonant donors, e.g., Ga. This fact shows that the conduction band shape is not modified. The substantially weak hybridization of conduction band states with the impurity $d$-states ( $s p-d$ hybridization), related to the incompatible symmetries of the states in question, means that the state of the mixed valence differs from that of the fluctuating valence system. Fe ions in $\mathrm{HgSe}$ form a state which may be regarded as an inhomogeneous mixed valence state.

\subsection{Electron mobility}

\subsubsection{Experiment}

The presence of the resonant state degenerate with the conduction band should strongly increase a scattering rate, i.e. reduce the electron mobility, specially at low temperatures in the mixed valence region of Fe donors. It should be caused by two mechanisms, the Kondo effect and the Friedel resonant scattering. Experimental data indicate to the opposite. Instead of an increase, the reduction of the scattering rate can be concluded for the mixed valence region. The electron mobility values are enhanced, and in the case of Shubnikov-de Haas experiments lowering of the Dingle temperature, $T_{\mathrm{D}}$, takes place (Dobrowolski et al. [11], Vaziri and Reifenberger [20]).

Figure 2 shows the dependence of the electron mobility at $4.2 \mathrm{~K}$ as a function of the Fe concentration. The experimental mobility data, in the mixed valence region, are greater by factor 4-5 than those of $\mathbf{H g S e}$ doped with non-resonant donors to a similar electron concentration. The effect is even more pronounced 


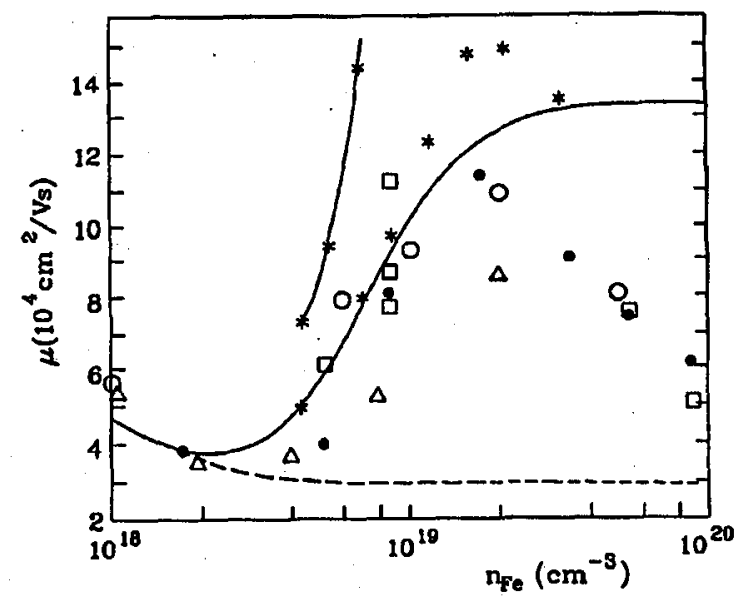

Fig. 2. Electron mobility at $4 \mathrm{~K}$ in $\mathrm{HgSe}: \mathrm{Fe}$ as a function of iron concentration. Experimental data are from: full circles [10], open circles [11], squares [87], triangles [27]. The broken line was calculated without the inter-donor Coulomb interaction, while the continuous curve includes this interaction in the short-range correlation model (after [34]). Asterisks joined with a full line represent the mobility values obtained from numerical simulations, similarly to those joined with a dotted line, which additionally include a contribution due to the resonant scattering [34] (Courtesy of Z. Wilamowski).

in the case of the Dingle temperature, the observed decrease covered an order of magnitude.

The analysis of the dependence of the electron mobility on the temperature shows that the reduction of the scattering rate is observable below, approximately, $100 \mathrm{~K}$ (Pool et al. [10]) where scattering by charged impurities is dominant.

Some important information on a behavior of the electron mobility in $\mathrm{HgSe}: \mathrm{Fe}$ was provided by experiments in the hydrostatic pressure (Dobrowolski et al. [26] and Skierbiszewski et al. [27]). An application of the hydrostatic pressure to HgSe causes a significant reduction of the energetic distance between $\Gamma_{6}$ light hole and $\Gamma_{8}$ conduction bands which, in turn, makes smaller the density of states in the conduction band. To compensate this effect either the Fermi level must be adjusted, if $n_{\mathrm{e}}$ is to remain constant $\left(n_{\mathrm{Fe}} \leq n_{\mathrm{Fe}}^{*}\right)$, or, if the Fermi level is pinned $\left(n_{\mathrm{Fe}}>n_{\mathrm{Fe}}^{*}\right)$, the concentration of the conduction electrons has to decrease. Thus, by applying the hydrostatic pressure to $\mathrm{HgSe}: \mathrm{Fe}$ in the mixed valence regime, one can vary the valence ratio $n_{\mathrm{Fe}^{2+}} / n_{\mathrm{Fe}^{3+}}\left(n_{\mathrm{e}}=n_{\mathrm{Fe}^{3+}}\right)$ in a sample in a reversible manner.

Figure 3 shows the mobility data in the investigated samples. Then the predictions of the standard theory (cf. Dietl and Szymanska [28]) assuming that the main scattering mechanism is that by charged donors (sufficient to describe the mobility in $\mathrm{HgSe}: \mathrm{Ga}$ ) is also shown. Considerable deviations of the experimental points from the standard theory in the case of the samples 2-5 are indicative of a reduction of the efficiency of the scattering by charged donors. The reduction is most pronounced for samples with the intermediate Fe concentrations (samples 3 and 4) and shows a tendency to a saturation in the sample with the highest 


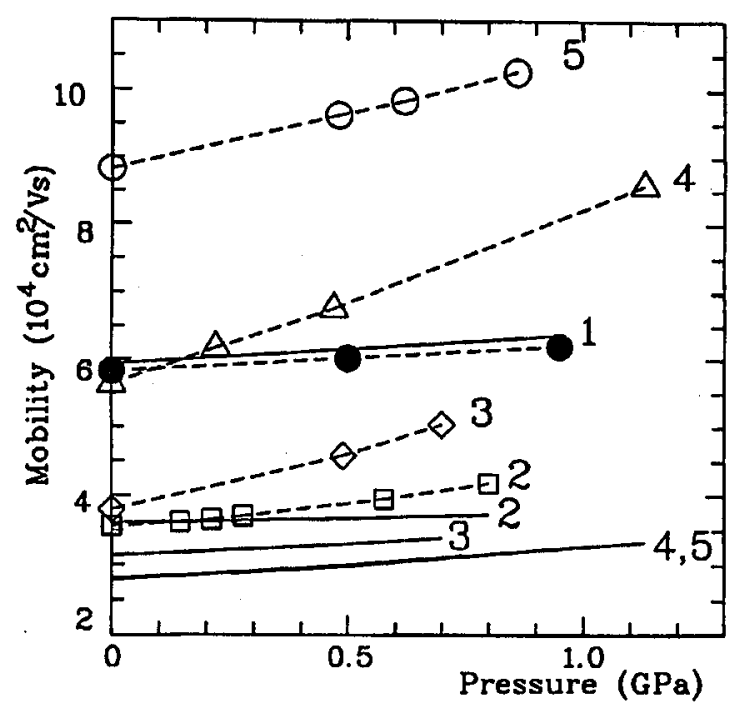

Fig. 3. Electron mobility in $\mathrm{HgSe}: \mathrm{Fe}$ as a function of hydrostatic pressure at $4.2 \mathrm{~K}$. Symbols represent experimental points and the solid lines are calculated assuming scattering by random ionized donors. The numerals correspond to the $n_{\mathrm{Fe}}, 1: 1 \times 10^{18} \mathrm{~cm}^{-3}$, 2: $2 \times 10^{18} \mathrm{~cm}^{-3}, 3: 4 \times 10^{18} \mathrm{~cm}^{-3}, 4: 8 \times 10^{18} \mathrm{~cm}^{-3}, 5: 2 \times 10^{19} \mathrm{~cm}^{-3}$ (after Ref. [26]).

Fe content. Sample 2 shows deviations from the theoretical curve starting only at a certain pressure, roughly corresponding to the pressure at which the electron concentration in this sample becomes pressure dependent (see Fig. 3). The temperature studies of the electron mobility under the hydrostatic pressure indicate that the enhancement becomes smaller at higher temperatures and disappears entirely at approximately $100 \mathrm{~K}$.

Because there was no indication of the Kondo effect or the Friedel resonant scattering in the $\mathrm{HgSe}: \mathrm{Fe}$ detected in the temperatures down to the temperature of pumped helium the investigations of the electron conductivity $\sigma$ in the millikelvin range were undertaken (Lenard et al. $[29,30]$ ). The electron conductivity in $\mathrm{HgSe}: \mathrm{Fe}$ samples was measured between $30 \mathrm{mK}$ and $4.2 \mathrm{~K}$. There were no significant changes of $\sigma$ detected in investigated samples. The weak temperature dependence indicates to the absence of the resonant scattering and the Kondo effect, even for quasiparticles having energies as low as $3 \mu \mathrm{eV}$. This provides evidence for the weak $s p-d$ hybridization.

In course of the investigation of the electron conductivity in the millikelvin temperature range the existence of a superconducting phase was detected in a few samples. The careful analysis of the experimental conditions allowed to find out that an appearance of the superconductivity is related to the presence of superconductive $\mathrm{Hg}$ inclusions. The effect appeared in samples grown with an excess of $\mathrm{Hg}$, and was not detected in samples grown from stoichiometric melts and in samples annealed in Se vapor. The annealing procedure is known to release an excess of $\mathrm{Hg}$ from the material. 


\subsubsection{Theoretical models}

A model exp'aining phenomena observed in $\mathrm{Hg}_{1-x} \mathrm{Fe}_{x} \mathrm{Se}$ was put forward by J. Mycielski [31]. The basic assumption of this model was that the resonant state was very narrow (i.e., long living). Additionally, the resonant state was assumed to be localized strongly enough to prevent formation of the impurity band. The model was based on an idea that in a system of resonant donors, with only a fraction of them being ionized, there was a possibility of an electron hop from one donor site to another. The existence of the Coulomb repulsion between ionized (i.e., empty) donors would force the hopping electrons to acquire (at low temperatures) such a spatial distribution which minimizes the total electrostatic energy. It would lead to a correlation of positions of charges localized on donors resulting from a tendency of an ionized donor to have as few charged donors in its proximit, as possible. Let us stress that the donors themselves do not change their positions, but it is the charge they trap that can move. By calculating the free energy of the system including the intersite screened Coulomb interaction Mycielski demonstrated that the most favorable arrangement of donor charges at low temperatures was, indeed, when the ionized donors were at furthest from each other. Moreover, he presented that the spatial ordering of the charged centers might lead to a "liquid state" of the system of charges on donors and, in the extreme case $n_{\mathrm{Fe}} \gg n_{\mathrm{Fe}}^{*}$, a nearly ordered structure - a Wigner crystal of localized electrons. An explicit calculation performed by J. Mycielski [31] showed that the energies of random $E_{\text {rd }}$ and ordered $E_{\text {latt }}$ networks of ionized Fe donors in $\mathrm{HgSe}$ differed by approximately $13 \mathrm{meV}$ per one ionized donor.

For small momentum transfers scattering of conduction electrons by charged centers whose positions are correlated is not very effective. This fact is responsible for the enhanced values of the mobility.

Dietl [32] pointed out that as a consequence of such correlation the so-called Coulomb gap (cf. Efros and Shklovskii [33]) in the one-particle density of donor states at the Fermi energy develops. The existence of the Coulomb gap, i.e. lack of impurity states at the Fermi energy (see Fig. 4), eliminates, otherwise expected, a strong reduction of the scattering rate due to the resonant scattering. According to Dietl, in $\mathrm{HgSe}: \mathrm{Fe}$, even if $s p-d$ hybridization is very weak, one should expect the mobility value about $20 \mathrm{~cm}^{2} /(\mathrm{V} \mathrm{s})$. The experimentally observed values ranged to $100000 \mathrm{~cm}^{2} /(\mathrm{V} \mathrm{s})$. This shows that the Coulomb gap in the impurity density of states function was formed.

In course of further studies the above presented ideas appeared to be qualitatively correct, although the range of the correlation of the position of charges was shown to be rather short. Taking explicitly into account screening of the inter-donor repulsion, Wilamowski et al. [34] performed calculation within an analytical short-range correlation model.

The short-range correlation model [34] assumes that at $T=0 \mathrm{~K}$ there exists a sphere with a radius $r_{c}$ centered on an ionized $\mathrm{Fe}^{3+}$ donor within which there are no other ionized donors. Only neutral, i.e., occupied by electrons, $\mathrm{Fe}^{2+}$ donors are present within the sphere. The above assumption is valid in the limit $1-n_{\mathrm{e}} / n_{\mathrm{Fe}} \ll 1$. Neglecting the correlation in the spatial positions of the ionized 


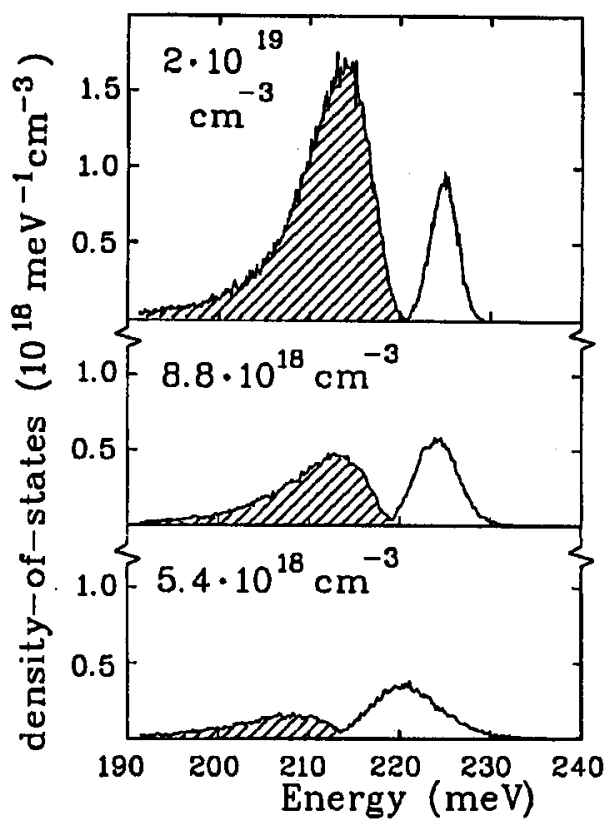

Fig. 4. One-particle density of Fe impurity states for three values of iron concentration obtained from numerical simulations. The bare energy of Fe impurity is at $210 \mathrm{meV}$. The occupied states are shown by the shaded area [34] (Courtesy of Z. Wilamowski).

donors which are more distant than $r_{c}$ the probability of finding ionized donors at a distance $r$ from the fixed one is given by the pair correlation function defined as

$$
g(r)= \begin{cases}0, & \text { for } r<r_{\mathrm{c}} \\ 1, & \text { for } r \geq r_{\mathrm{c}}\end{cases}
$$

For small values of $1-n_{\mathrm{e}} / n_{\mathrm{Fe}}$ holds a relation

$$
n_{\mathrm{e}} V_{\mathrm{c}}=1-\exp \left(-n_{\mathrm{Fe}} V_{\mathrm{c}}\right) \text {, }
$$

where $V_{c}=4 \pi r_{c}^{3} / 3$. This equation determines $r_{c}$ and, thus, $g(r)$. This value can be used in turn, to calculate the structure factor

$$
S(q)=1+4 \pi n_{\mathrm{e}} \int\{[g(r)-1] \sin (q r) / q\} \mathrm{d} r .
$$

The momentum relaxation time, for the scattering by the ionized donors, may be expressed in terms of $S(q)$ :

$$
\tau^{-1}=\frac{m^{*} n}{4 \pi \hbar^{3} k^{3}} \int_{0}^{2 k_{\mathrm{F}}}|V(q)|^{2} S(q) q^{3} \mathrm{~d} q,
$$

where $V(q)$ is the matrix element of the screened Coulomb potential due to a single impurity, which for zinc-blende narrow gap semiconductors is given by

$$
|V(q)|^{2}=\left[\frac{4 \pi e^{2}}{\kappa_{0} q^{2}\left(1+\frac{1}{\lambda^{2} q^{2}}\right)}\right]\left(1+\frac{q^{2}}{k^{2}} A+\frac{q^{4}}{4 k^{4}} B\right),
$$


where $\lambda$ is a screening length of the band electrons,

$$
A=b^{2}\left(\frac{1}{2} b-\sqrt{2} c\right)^{2}-b^{2}-c^{2}
$$

and

$$
B=\left(b^{2}+c^{2}\right)^{2}-b^{2}\left(\frac{1}{2} b-\sqrt{2} c\right) .
$$

Coefficients $b$ and $c$ denote the contribution of the $p$-type component in the Bloch wave function

$$
\begin{aligned}
& b^{2}=\frac{\Delta^{2}}{3 D}\left(E-E_{0}\right), \\
& c^{2}=\frac{2}{3 D}\left(\Delta+\frac{3}{2} E\right)^{2}\left(E-E_{0}\right)
\end{aligned}
$$

and

$$
D=\left(\Delta+\frac{3}{2} E\right)(\Delta+E) E+(\Delta+E)^{2}\left(E-E_{0}\right)+\frac{1}{2}\left(E-E_{0}\right) E^{2} .
$$

$E$ is the solution of the Kane equation [35] for the conduction band

$$
E+(E+\Delta)\left(E-E_{0}\right)-k^{2} P^{2}(E+2 / 3 \Delta)=0 .
$$

The zero of energy scale is at the top of the valence band. The $E_{0}$ is the $\Gamma_{6}-\Gamma_{8}$ energy gap (which in the case of inverted band type structure materials is negative), $P$ is the $s-p$ momentum matrix element, and $\Delta$ is the energy of spin-orbit splitting.

The electron lifetime for the scattering by the ionized donors is given by an expression analogous to Eq. (6) with the power of $q$ in the integrand reduced from 3 to 1 . It may be noted that the lifetime is even more susceptible to a modification of $S(q)$ due to the correlation since it is sensitive also to the small angle scattering, while the momentum relaxation time is determined mainly by the large angle scattering.

Within the short-range correlation model it is also possible to calculate the contribution $E_{\mathrm{c}}$, due to the Coulomb interaction, to the total energy of the system consisting of band and donor electrons. The minimum of the total energy determines an equilibrium concentration of electrons in the conduction band and it determines the Fermi energy.

The short-range correlation model describes adequately various phenomena observed in $\mathrm{HgSe}: \mathrm{Fe}$ : the dependence of $n_{\mathrm{e}}$ on $n_{\mathrm{Fe}}$ and the enhancement of the mobility. It explains also results of experiments performed under hydrostatic pressure which made possible reaching the mixed valence regime in the samples doped to $n_{\mathrm{Fe}}<n_{\mathrm{Fe}}^{*}$. Figure 5 shows the comparison of a short-range correlation model prediction compared with the experimental changes of the electron mobility and the electron concentration on the $\mathrm{Fe}$ concentration in $\mathrm{HgSe}: \mathrm{Fe}$ under the hydrostatic pressure of $7 \mathrm{kbar}$.

The Monte Carlo study of the system of partially filled resonant impurities, performed by Wilamowski et al. [34], showed that the short-range correlation model was qualitatively in agreement with numerical simulations. In the latter 


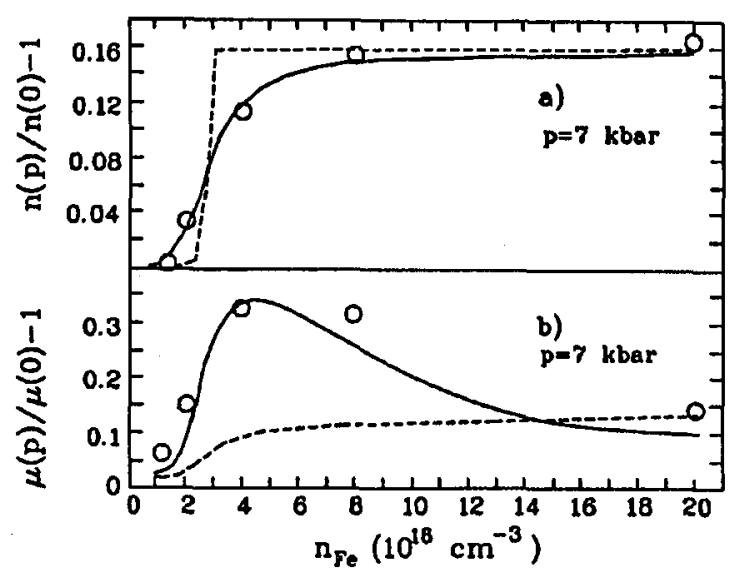

Fig. 5. Changes induced by the hydrostatic pressure of $7 \mathrm{kbar}$ in (a) the electron concentration and (b) the electron mobility in $\mathrm{HgSe}: \mathrm{Fe}$ at $4.2 \mathrm{~K}$. The experimental points are from [27]. The broken lines are calculated without the inter-donor repulsion, while the full curves account for the interaction within the short-range correlation model [34] (Courtesy of Z. Wilamowski).

studies the Coulomb energy gap was shown to be by at most $10 \%$ greater than that calculated analytically within the short-range correlation model. However, the electron mobility calculated using $g(r)$ obtained from the Monte Carlo simulation appeared to be considerably greater than that evaluated from the short-range correlation model. These results reflect the fact that in the numerical simulation of the pair correlation function some elements of long-range correlations were present which strongly affected the scattering rate. Both approaches mentioned above were descriptions basically for $T=0$.

The short-rane correlation model was extended by Tsidil'kovskir et al. [36]. By applying methods of statistical physics the authors were able to describe the dependence of the electron mobility on the temperature and on the magnetic field, in an extended region of $n_{\mathrm{Fe}}$.

\subsubsection{Spin-disorder and alloy (chemical disorder) scattering}

The presence in the host material of foreign atoms possessing the magnetic moments leads to additional channel of scattering. The interaction describing the coupling of conduction electrons and.magnetic impurities may be described by Hamiltonian (Kossut [37]):

$$
\mathcal{H}_{i}=\sum_{n} V\left(r-R_{n}\right)-\sum_{n} I\left(r-R_{n}\right) \sigma S_{n},
$$

where $R_{n}$ denotes the position of a magnetic impurity, $V\left(r-R_{n}\right)$ describes the spin-independent part of the interaction, and $I\left(r-R_{n}\right)$ is an exchange term.

For magnetic field weak enough (i.e. when the redistribution of electrons between spin sublevels may be neglected) the second process may be regarded as elastic. The first process, which is due to spin-independent part, is also elastic.

The electron mobility $\mu$ in the case of the strong degeneracy of electrons for 
spin-independent part (alloy scattering) may be written in a form (Kossut [38]):

$$
\mu=\mu_{0} k^{-3}\left(\frac{\mathrm{d} \epsilon}{\mathrm{d} k}\right)^{2} \frac{1}{F_{\mathrm{d}}}
$$

where

$$
\mu_{0}=\frac{\pi e}{\hbar x(1-x) N V^{2}}
$$

and

$$
F_{\mathrm{d}}=a^{4}+\left(\frac{W}{V}\right)^{2}\left[\frac{1}{3}\left(c^{2}+b^{2}\right)^{2}+\frac{2}{3} b^{2}\left(\frac{b}{2}-2 c\right)^{2}\right]-\frac{2}{3} \frac{W}{V} a^{2}\left(c^{2}+b^{2}\right)
$$

where $a$, which represents a contribution of $s$-type component in the Bloch function, is given by

$$
a^{2}=\frac{1}{D}\left(\Delta+\frac{3}{2} E\right)(\Delta+E) E,
$$

and $b, c$ and $D$ are given by Eqs. (10), (11) and (12) respectively. The matrix elements $V$ and $W$ are defined by

$$
V=\left\langle S\left|V_{a b}(r)\right| S\right\rangle
$$

and

$$
W=\left\langle X\left|V_{a b}(r)\right| X\right\rangle .
$$

$S, X, Y, Z$ are the Kohn-Luttinger amplitudes of the electron wave functions which transform like $s$ - and $p_{x^{-}}, p_{y^{-}}, p_{z}$-functions under operations of the $T_{d}$ point group. $W$ expresses the energy distance in the position of the $\Gamma_{8}$ band edges for end point materials and $V$ is an analogous distance for $\Gamma_{6}$ bands.

The electron mobility $\mu$ for spin-dependent scattering in the case of the strong degeneracy of electrons can be written in a form

$$
\mu=\mu_{0} k^{-3}\left(\frac{\mathrm{d} \epsilon}{\mathrm{d} k}\right)^{2} \frac{1}{F_{\mathrm{d}}},
$$

where

$$
\mu_{0}=\frac{\pi e}{\hbar x N \alpha^{2}},
$$

and

$$
\begin{aligned}
F_{\mathrm{d}}= & \left\{2 a^{4}+\left(\frac{\beta}{\alpha}\right)^{2}\left[\frac{2}{3}\left(c^{4}+b^{4}\right)+\frac{5}{3} c^{2} b^{2}+\frac{\sqrt{2}}{3} b^{3} c\right]\right. \\
& \left.-\frac{2}{3} \frac{\beta}{\alpha} a^{2}\left(-b^{2}+2 c^{2}\right)\right\} \frac{S(S+1)}{12} .
\end{aligned}
$$

The $s-d$ and $p-d$ exchange integrals $\alpha$ and $\beta$ are defined by

$$
\alpha=\langle S|I(r)| S\rangle,
$$

and

$$
\beta=\langle X|I(r)| X\rangle .
$$

While the second of the described mechanisms, the spin-dependent scattering, is weak and has never been taken into account in describing transport 
properties of $\mathrm{HgSe}: \mathrm{Fe}$, the first one, the alloy scattering, occurred to be important in describing the dependence of the electron mobility on composition $x$ in $\mathrm{Hg}_{1-x} \mathrm{Fe}_{x} \mathrm{Se}$ mixed crystals. The observed mobility drop for $\mathrm{Fe}$ molar fraction $x>0.02$ (see Fig. 2) was explained as being due to the alloy scattering (Skierbiszewski et al. [39]). As a result of the fitting procedure $W$ and $V$ matrix elements were determined as $W=-0.4 \mathrm{eV}$ and $V=3.3 \mathrm{eV}$.

From the presented above equations the electron lifetimes for the spin disorder and the chemical disorder scattering processes may be deduced, provided that the term proportional to the $W \times V$ in Eq. (17) and term proportional to $\alpha \times \beta$ in Eq. (23) are omitted.

\subsection{Thermomagnetic effects}

Among many results supporting the model presented in the previous section those published by Kuleev et al. [40] are of a special significance. The longitudinal and the transverse Nernst-Ettingshausen effects are very sensitive to the nature of carriers scattering. This is because they do not vanish only on account of energy dependence of the relaxation time. The reported by Kuleev [40] investigation of Nernst-Ettingshausen effect were performed in temperatures $9-40 \mathrm{~K}$ on $\mathrm{HgSe}$ samples doped with $F e$ to the concentration $1 \times 10^{18} \leq n_{\mathrm{Fe}} \leq 4 \times 10^{20} \mathrm{~cm}^{-3}$. The experimental results of transverse Nernst-Ettingshausen effect data plotted against the $F e$ concentration show that the effect changes its sign from negative to the positive one when the Fe concentration in samples becomes greater than $4.5 \times 10^{18} \mathrm{~cm}^{-3}$. This is a direct evidence for the change of the scattering mechanism. The results obtained are consistent with the short-range correlation model predictions. When the $\mathrm{Fe}^{3+}$ system becomes spatially correlated the sign of Nernst-Ettingshausen effect changes to the opposite due to the change of the dependence of the relaxation time on the energy.

\subsection{Optical properties}

At early stages of studying of $\mathrm{HgSe}: \mathrm{Fe}$ and related compounds optical methods had played minor role, as compared to the transport ones. This fact resulted from difficulties in investigating materials with the very high electron concentration. Nevertheless, it should be noted that a manifestation of unusual properties of $\mathrm{Hg}_{1-x} \mathrm{Fe}_{x} \mathrm{Se}$ was for the first time reported in 1981 by Serre et al. [41] on the basis of interband magnetooptical studies. The authors observed strong oscillations of magnetotransmission signal instead of the expected resonances due to interband transitions. In succeeding years qualitatively similar oscillations were observed in the strip-line experiments (Portugall et al. [23, 42] and Buchholz-Stepputtis et al. $[24,25])$. Their origin is however not yet fully understood and the nature of these oscillations should be a subject of further investigations (cf. von Ortenberg [43]).

Szuszkiewicz et al. [14] performed optical transmission and reflection experiments on $\mathrm{Hg}_{1-x} \mathrm{Fe}_{x} \mathrm{Se}$ samples. The experimental curves were compared with the theoretical one, calculated using the formalism of the dynamic dielectric function with the Drude-Zener free-carrier contributions. It was deduced that the Fermi level is pinned to the $\mathrm{Fe}^{2+}$ state which, at helium temperatures, is not higher than 
$200 \mathrm{meV}$ above the bottom of the conduction band. This result fully confirms the earlier estimations which were based on the transport measurements. A few papers (Szuszkiewicz et al. [44] and references therein) were devoted to study relaxation time in the far infrared frequency range. The authors employed the fact that the plasmon related free-carrier absorption is sensitive to small momentum transfers. In the paper [45] the experimental results are compared with the finite temperature Monte Carlo simulations. It is shown that the correlation of charged centers exists in a wide temperature range (it is shown that effects due to correlations exist even at room temperature!).

Because of the very high carrier concentration in $\mathrm{Hg}_{1-x} \mathrm{Fe}_{x} \mathrm{Se}$ no optical or magnetooptical transmission experiment for radiation below the plasma energy $\hbar \omega \approx 87 \mathrm{meV}$ is possible. Thus to investigate intraband magnetooptical transitions one has to shift their energies into a range higher than this value. The last may be achieved by applying extremely high magnetic field, close to mega-Gauss region. The results of such experiment performed in magnetic fields up to $150 \mathrm{~T}$ were presented by Portugall et al. [23]. The experiment was carried out using the destructive coil technique with the pulse duration of some microseconds. The light source was the $\mathrm{CO}_{2}$ laser. The recorded spectra exhibited a multi-line structure. The authors interpret the observed lines as a cyclotron resonance and its higher harmonics. The effective mass value was found to be $m_{c}=0.056 m_{0}$. This value is about $10 \%$ smaller than those known from other investigations. It is likely due to influencing the spectra by hot carrier transition. The hot carriers are generated in $\mathrm{HgSe}$ - a strongly conducting material - by a strong electric field generated within a sample by a magnetic field pulse.

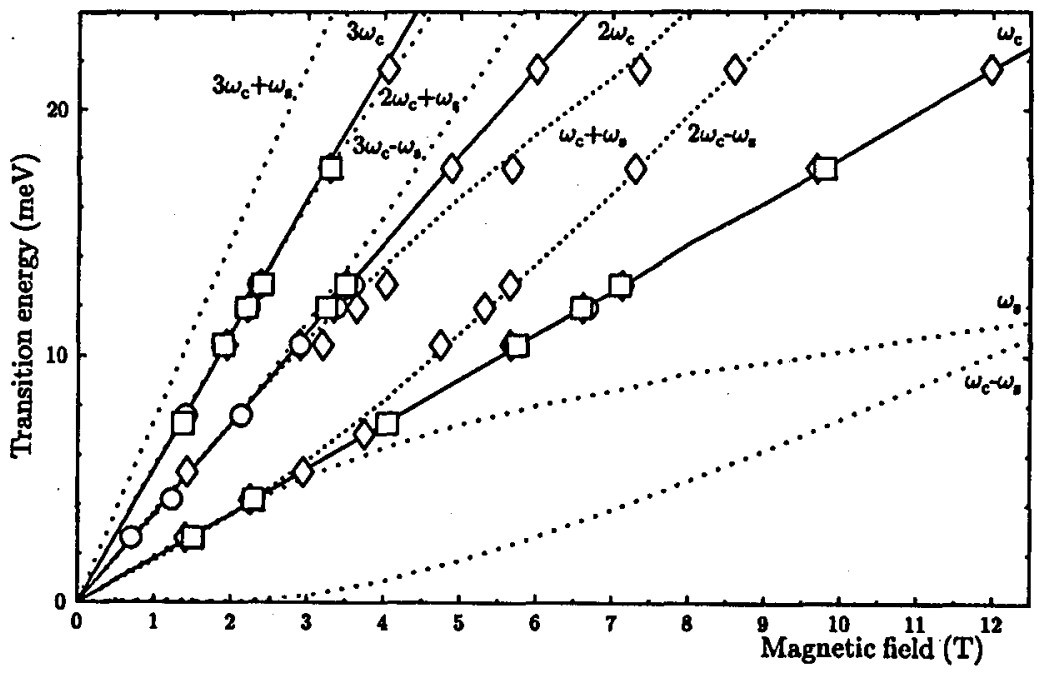

Fig. 6. Strip-line spectra resonance position in HgSe:Fe sample $\left(n_{\mathrm{Fe}}=1 \times 10^{20} \mathrm{~cm}^{-3}\right)$ (after Ref. [25]). Solid lines represent theory and symbols - experiment performed in different strip-line configurations: diamonds - parallel, circle - perpendicular and squares - surface. 
Recently, careful investigations of IIgSe:Fe samples in a multi-reflection experiment achieved by the strip-line technique were reported by Buchholtz-Stepputtis [25]. The obtained spectra, besides the fundamental cyclotron resonance and combined spin flip resonances showed also the second and third harmonics of cyclotron resonance transitions. The position of the resonances vs. magnetic field are presented in Fig. 6. The analysis of experimental data yielded a precise value of the cyclotron mass $m_{\mathrm{c}}=(0.064 \pm 0.002) m_{0}$. This value is, again, in agreement with those obtained from transport (Shubnikov-de Haas) data, contrary to the reported above direct cyclotron resonance measurements performed in pulsed magnetic field.

\subsection{Magnetic properties}

The presence of iron in two charge states, namely $\mathrm{Fe}^{2+}$ and $\mathrm{Fe}^{3+}$ in $\mathrm{Hg}_{1-x} \mathrm{Fe}_{x} \mathrm{Se}$ makes magnetic properties of this crystal very interesting. $\mathrm{Fe}^{2+}$ and $\mathrm{Fe}^{3+}$ display entirely different properties. The ground state of the $\mathrm{Fe}^{2+}(S=2$, $L=2$ ) in the tetrahedral environment is a magnetically inactive singlet. Therefore system of $\mathrm{Fe}^{2+}$ ions behaves in a manner characteristic of Van Vleck paramagnet. The $\mathrm{Fe}^{3+}$ ions $(S=5 / 2, L=0)$ bring in, to the magnetic properties of the material under study, the Curie-like paramagnetic contribution. For sufficiently large content $x$ of $\mathrm{Fe}$, effects connected with antiferromagnetic coupling between magnetic ions become nonnegligible.

The first investigation of magnetic properties of $\mathrm{Ig}_{1-x} \mathrm{Fe}_{x} \mathrm{Se}(0.05 \leq x \leq 0.1)$ was carried out by Lewicki et al. [46]. As a result of magnetic susceptibility and specific heat studies the exchange integral $2 J / k_{\mathbf{B}}$ describing the total exchange energy of a nearest-neighboring pair of $\mathrm{Fe}^{2+}$ ions was established to be approximately $30 \mathrm{~K}$.

The detailed investigations of samples with the low iron content $(x<0.01)$ were performed by Arciszewska et al. [47-49]. In her papers it was explicitly demonstrated that the temperature dependence of magnetic susceptibility may be explained in terms of Van Vleck susceptibility of $\mathrm{Fe}^{2+}$ ions superimposed on the Curie-like susceptibility of $\mathrm{Fe}^{3+}$ ions. Figure 7 shows the temperature variation of magnetic susceptibility in $\mathrm{Igg}_{1-x} \mathrm{Fe}_{x} \mathrm{Se}$, and for comparison in $\mathrm{Hg}_{1-x} \mathrm{Mn}_{x} \mathrm{Se}$ at low temperatures after Ref. [49].

The magnetization study of $\mathrm{Hg}_{1-x} \mathrm{Fe}_{x}$ Se was performed by von Ortenberg et al. [50], Wilamowski et al. [51], Dybko et al. [52] and Zeitler et al. [53]. The very characteristic feature observed at low temperatures is the presence of de Haasvan Alphen oscillations superimposed on the magnetization curve. This not very common behavior results from anomalously high amplitude of the de Haasvan Alphen oscillations, which, in turn, results from very low values of Dingle temperature - a symptom of the space correlation of charged impurities.

Analyzing results of magnetic susceptibility, high field torque and magnetization Wilamowski et al. [51] concluded that the energy splitting of the Fe levels in $\mathrm{HgSe}$ is substantially larger than in other selenides $(\approx 2$ times). In consequence, the magnetic susceptibility in $\mathrm{Ig}_{1-x} \mathrm{Fe}_{x} \mathrm{~S}$ achieves values lower than in other materials. 


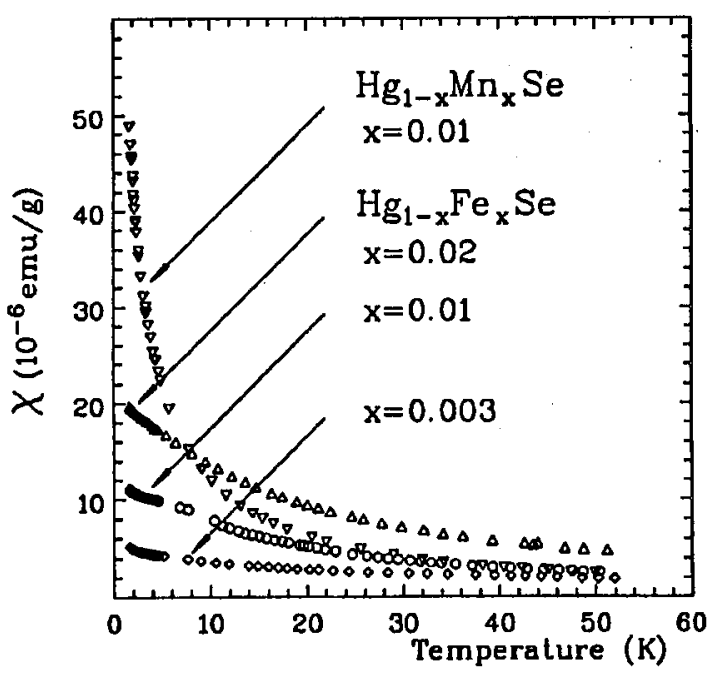

Fig. 7. Magnetic susceptibility as a function of temperature in $\mathrm{Hg}_{1-x} \mathrm{Fe}_{x} \mathrm{Se}$ and $\mathrm{Hg}_{1-x} \mathrm{Mn}_{x}$ Se. The weak dependence of magnetic susceptibility observed for $\mathrm{Fe}$ in $\mathrm{HgSe}$ indicates that in this case we do not deal with pure Van Vleck paramagnetism (after Ref. [47]).

Recently Dybko et al. [52] reported the magnetization study of strongly diluted $(x \leq 0.003) \mathrm{Hg}_{1-x} \mathrm{Fe}_{x}$ Se samples. The authors showed that the appropriate theoretical models describe well the magnetization in samples containing isolated $\mathrm{Fe}^{3+}(x=0.002)$ or $\mathrm{Fe}^{2+}$ ions ${ }^{\dagger}$. Contrary to the expectation, the magnetization in the sample containing iron in both charge states i.e. $\mathrm{Fe}^{3+}$ and $\mathrm{Fe}^{2+}(x=0.003)$ appeared to be not a simple superposition of the contributions arising from the presence of the two magnetic subsystems. According to the authors this result may suggest an existence of an interaction between $\mathrm{Fe}^{3+}$ and $\mathrm{Fe}^{2+}$ magnetic subsystem, the interaction which is extremely sensitive to the number of each species. The results of Ref. [52] indicate that further experimental and theoretical efforts should be undertaken to understand fully magnetic properties of $\mathrm{Hg}_{1-x} \mathrm{Fe}_{x} \mathrm{Se}$.

\section{HgSe based mixed crystals doped with Fe}

A look at the localization of Fe level in different II-VI compounds allows us to see some tendencies present in its energy position (e.g. [8]). This indicates to the possibility that the energy of $\mathrm{Fe}^{2+}$ state $E_{\mathrm{Fe}}$ may be varied in mixed compounds by replacing some of Se ions by foreign ions. In such way it would be possible to control parameters which determine the mixed valence regime, in particular, the ratio of concentrations of ionized to neutral donors. Additionally, the Fe doping would result in obtaining samples with a high mobility and, thus, a low Dingle temperature or, more precisely, a short lifetime of an electron on a given Landau-quantized state.

\footnotetext{
${ }^{\dagger}$ The sample containing only $\mathrm{Fe}^{2+}$ ions was grown by simultaneous doping of $\mathrm{HgSe}$ with $\mathrm{Fe}$ and $\mathrm{Ga}$.
} 
Presently there are available data concerning five mixed systems: two containing wide gap II-VI semiconductors: CdSe (Mycielski et al. [9], Wolff et al. [54]) and $\mathrm{ZnSe}$ (Dobrowolski et al. [55, 56]), the other two with narrow gap II-VI semiconductors HgTe (Dobrowolski et al. [26]) and $\beta$-HgS (Szuszkiewicz et al. [57, 58], Dybko [59]) and the last one with magnetic semiconductor MnSe (Dobrowolski et al. [60,62, 56], von Ortenberg et al. [61]) as a component. The solid solutions of CdSe and $\mathrm{HgSe}$ may be obtained in zinc-blende structure up to $x=0.7$ and in wurtzite structure above $x=0.8$, the one-phase zinc-blende structure mixed crystals may be grown in the full composition range within the next three systems (Crucheanu et al. [63] and Giriat [64]), whereas the MnSe forms solid solutions with $\mathrm{HgSe}$ up to 38 at.\% only (Gałazka and Kossut [65]).

$$
\text { 3.1. } H g_{1-x} M n_{x} S e: F e
$$

Samples with low Dingle temperatures are vital when one's object is to study quantum oscillatory phenomena in materials with a high electron concentration. That is often the case of semimagnetic semiconductors, in which direct observation of the spin splitting is crucial. Usually when a high concentration is achieved by doping with other impurities, for instance $\mathrm{Ga}$, In, the charged centers scattering results in a relatively low mobility values (high Dingle temperatures), making observation of the spin splitting of conduction band states impossible. The verification of the above hypothesis was the main goal which led to investigations of $\mathrm{Hg}_{1-x} \mathrm{Mn}_{x} \mathrm{Se}$ (Dobrowolski et al. [56, 60,62] and von Ortenberg et al. [61]).

Electronic and magnetic properties of semimagnetic semiconductors $\mathrm{Hg}_{1-x} \mathrm{Mn}_{x} \mathrm{Se}$ are well established (cf. Kossut and Dobrowolski [4]). The triple degeneracy point, i.e., $\mathrm{Mn}$ content at which the bands $\Gamma_{6}$ and $\Gamma_{8}$ are degenerate, occurs at 6.2 at.\% of $\mathrm{Mn}$ at $4.2 \mathrm{~K}$. The exchange constants between the mobile electrons and the localized on $\mathrm{Mn}$ ions $3 d$ electrons were found to be $N_{0} \alpha=-0.4 \pm 0.1 \mathrm{eV}$ and $N_{0} \beta=0.7 \pm 0.1 \mathrm{eV}$ where $N_{0}$ is the volume of the unit cell. The momentum matrix element $P$ decreases with an increasing Mn molar fraction $x$ from $P=7.2 \times 10^{-8} \mathrm{eV}$ cm for IgSe to $P=5.9 \times 10^{-8} \mathrm{eV} \mathrm{cm}$ for $x=0.115$. The spin-orbit splitting $\Delta$ and the higher band parameters are composition and temperature independent and their values are the same as in pure $\mathrm{HgSe}$ (Dobrowolska et al. [66]).

A qualitative analysis of the results of the investigations of iron doped $\mathrm{Hg}_{1-x} \mathrm{Mn}_{x} \mathrm{Se}$ (Dobrowolski et al. [56]) indicates that there exist close similarities between the properties of the investigated materials and those of HgSe:Fe. Figure 8 shows the dependence of the electron concentration on the iron doping level at liquid helium temperature for $x \approx 0.0025$ and $x \approx 0.04$. The initial increase in the electron concentration in proportion to the amount of $\mathrm{Fe}$ is followed by a plateau where the electron concentration does not depend on the amount of iron. The height of the plateau decreases with the Mn molar content $x$ which indicates that a relative shift of the iron state energy and the bottom of the conduction band occurs when the composition of the crystal changes. The electron concentration plotted as a function of temperature also resembles that observed previously in $\mathrm{HgSe}: \mathrm{Fe}$ (Pool et al. [10]). Namely, in the heavily doped samples $\left(n_{\mathrm{Fe}} \geq\right.$ $5 \times 10^{19} \mathrm{~cm}^{-3}$ ) an increase in $n_{\mathrm{e}}$ with increase in temperature $T$ is observed, while 


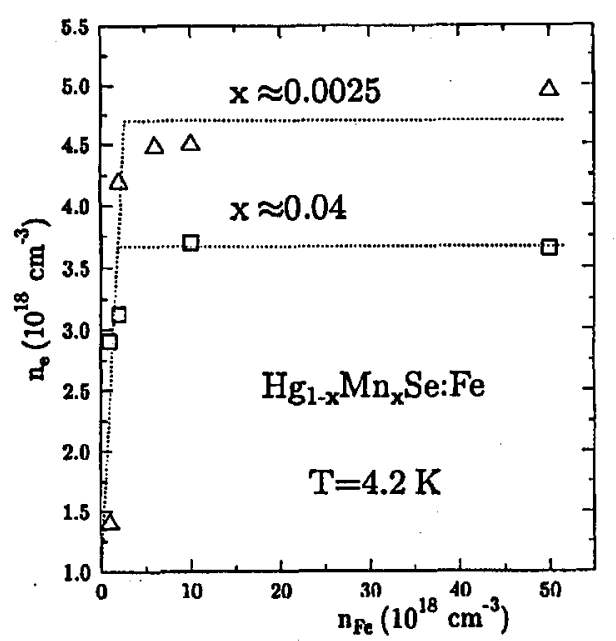

Fig. 8. The dependence of the electron concentration in $\mathrm{Hg}_{1-x} \mathrm{Mn}_{x} \mathrm{Se}$ :Fe for $x \approx 0.0025$ and $x \approx 0.04$ on iron doping at $4.2 \mathrm{~K}$. The lines are to guide the eye (after Ref. [56]).

in samples with fewer Fe impurities the electron concentration is nearly constant or it even decreases slightly.

Also results of the Shubnikov-de Haas effect measurements show similarities to that for HgSe:Fe. Figure 9 presents an oscillatory part of the transverse magnetoresistance for two samples with the Mn mole fraction $x=0.0025$, doped with iron to the quantity $n_{\mathrm{Fe}}=2 \times 10^{18} \mathrm{~cm}^{-3}$ and $n_{\mathrm{Fe}}=1 \times 10^{19} \mathrm{~cm}^{-3}$, respectively. The second sample shows more pronounced oscillations, which indicate that the Dingle temperature for this sample is substantially lower (in contrast to normally expected increase in $T_{\mathrm{D}}$ with growing $n_{\mathrm{e}}$ ).

Apart from similarities, it is also evident from the mobility and the Dingle temperature data that the reduction of the scattering rate is smaller in $\mathrm{Hg}_{1-x} \mathrm{Mn}_{x}$ Se than in HgSe:Fe. This is because of the additional scattering mechanisms which contribute to the total relaxation time in mixed crystals with $\mathrm{Mn}$.

The procedure of the calculation of the theoretical mobility and the Dingle temperature accounted for the scattering by charged impurities (with the reduction due to the spatial correlation), the alloy scattering, and the spin-dependent scattering (scattering by magnetic moments localized on Mn ions). The spin-dependent scattering by the magnetic moments localized on $\mathrm{Fe}$ ions was neglected, since their number is at least by a factor of 10 smaller than those localized on Mn. It is to be noted that in samples with the higher values of the mole fraction $x$ the reduction of the scattering rate is less prominent because of an increasing influence of the alloy scattering on the total electron mobility or the Dingle temperature.

Figure 10 shows the dependence of the electron mobility on iron doping level for the samples with Mn content $x=0.0025$. The enhancement of the mobility in the mixed valence regime, i.e., for $n_{\mathrm{Fe}}>n_{\mathrm{Fe}}^{*}$ is evident. A similar dependence for the Dingle temperature is presented in Fig. 11.

In both cases the discrepancy between the calculations and the experiment 


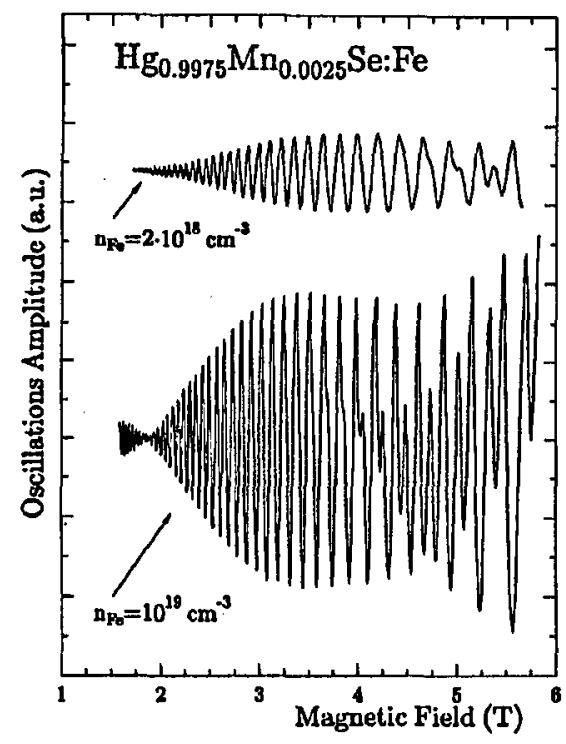

Fig. 9. Oscillatory part of the magnetoresistance recorded at $4.2 \mathrm{~K}$ for two $\mathrm{Hg}_{1-x} \mathrm{Mn}_{x}$ Se samples: doped with (a) $2 \times 10^{18} \mathrm{~cm}^{-3} \mathrm{Fe}$, and (b) $1 \times 10^{19} \mathrm{~cm}^{-3} \mathrm{Fe}$ (after Ref. [56]).

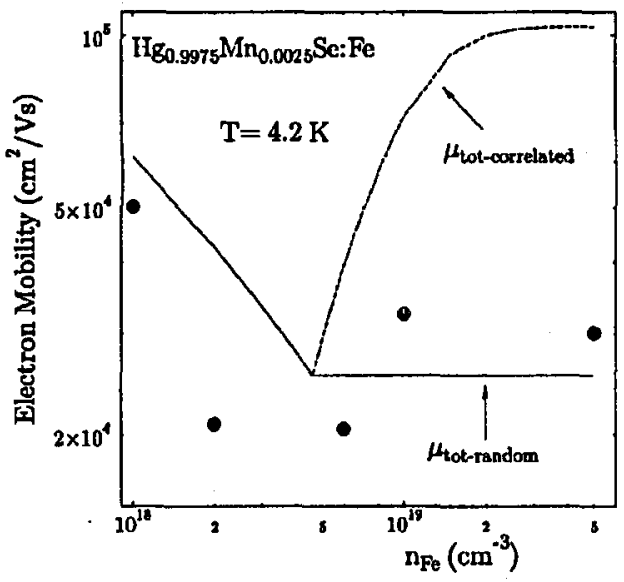

Fig. 10. The electron mobility in $\mathrm{Hg}_{0.9975} \mathrm{Mn}_{0.0025} \mathrm{Se}: \mathrm{Fe}$ vs. iron concentration. The solid line represents the theory for uncorrelated charged centers system, dashed for correlated system, full circles - experiment (after Ref. [56]).

grows with the increasing iron doping - this may be related to an increasing role of the resonant scattering not included in the theoretical calculation. Because of the existence of the Coulomb gap this mechanism is to a great extent reduced, nevertheless it does exist and itsinfluence is expected to grow with the growing chemical 


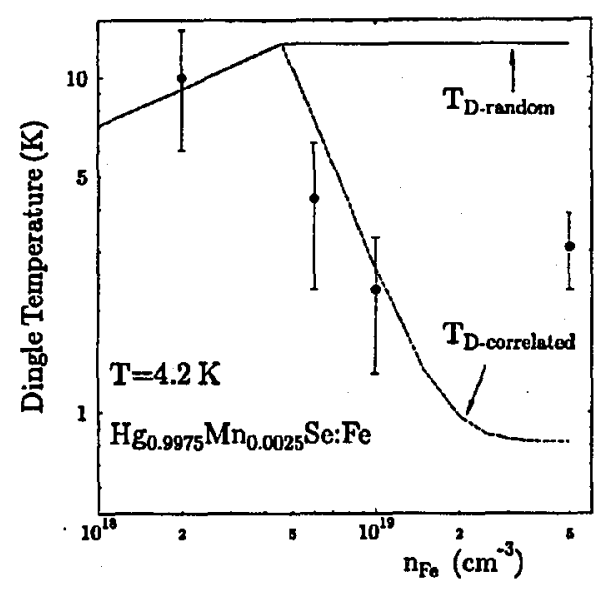

Fig. 11. The Dingle temperature for $\mathrm{Hg}_{0.9975} \mathrm{Mn}_{0.0025} \mathrm{Se}: \mathrm{Fe}$ vs. iron concentration. The solid line - theory for uncorrelated charged centers system, dashed for correlated system, full circles - experiment (after Ref. [56]).

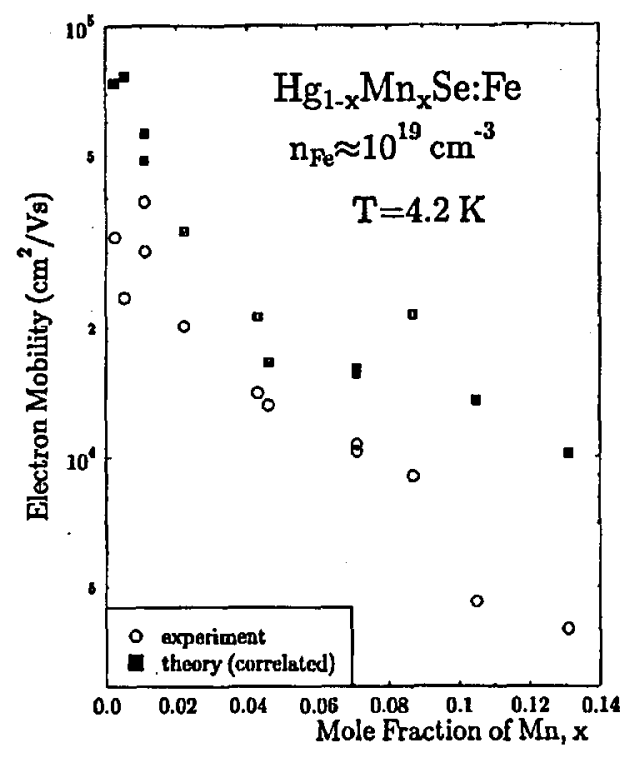

Fig. 12. The electron mobility in $\mathrm{Hg}_{1-x} \mathrm{Mn}_{x} \mathrm{Se}: 10^{19} \mathrm{~cm}^{-3}$ Fe for samples from the mixed valence regime (after Ref. [56]).

disorder in the material, since then the Coulomb gap gradually disappears.

The dependence of the electron mobility on the Mn mole fraction $x$, for samples in the mixed valence regime (i.e., $n_{\mathrm{Fe}}>n_{\mathrm{Fe}}^{*}$ ), is presented in Fig. 12. Similarly to the case of $\mu$ vs. $n_{\mathrm{Fe}}$ dependence - also here the discrepancy between the measured and the calculated mobility values grows with increasing $x$. The 
reason is very likely the same - an increasing role of the resonant scattering with a growing disorder in the sample. Similarly some discrepancies between theory prediction and experiments were observed in the case of the Dingle temperature.

The constant value of the electron concentration as a function of iron concentration in the mixed valence regime (where pinning of the Fermi level to the iron related impurity level occurs) enables the determination of the energy of the donor level. Assuming that the Fermi level in these samples is pinned to the resonant donor related state and making use of the phenomenological Langer and Heinrich rule [8], which states that the energy of the iron related level is constant on the absolute energy scale, the energy position of Fe level was determined as a function of Mn molar fraction $x$. This in turn enables to estimate the conduction and the valence band offsets defined as $V=E_{\Gamma_{6}}(\mathrm{MnSe})-E_{\Gamma_{6}}(\mathrm{HgSe})=3 \pm 1 \mathrm{eV}$, $W=E_{\Gamma_{8}}(\mathrm{MnSe})-E_{\Gamma_{8}}(\mathrm{HgSe})=-1.2 \pm 0.5 \mathrm{eV}$ respectively. The evaluated parameters $W$ and $V$ are directly related with the strength of the alloy scattering (cf. Eq. (15)) - one of the main mechanisms limiting the mobility in narrow gap $n$-type mixed crystals at low temperatures.

Summarizing the results presented above it may be noted that the reduction factor of the scattering rate in $\mathrm{HgSe}: \mathrm{Fe}$ differs from that in $\mathrm{Hg}_{1-x} \mathrm{Mn}_{x} \mathrm{Se}: \mathrm{Fe}$. The reason for that are the contributions originating in additional scattering mechanisms, namely the alloy and the spin-dependent scattering. Additionally, the effect of the disorder introduced by the presence of the four types of the atoms constituting the crystal can lead to a smearing off the resonance in the density of states function and to an impairment of the Coulomb gap. Thus the reduction of the resonant scattering related to the existence of the Coulomb gap loses its effectiveness and this type of scattering may become appreciable again.

$$
\text { 3.2. } H g_{1-v} C d_{v} S e: F e
$$

$\mathrm{Hg}_{1-v} \mathrm{Cd}_{v} \mathrm{Se}$ is an example of a compound in which upon replacing a part of mercury atoms with foreign atoms (cadmium) the spacing between the bands of $s$ and $p$ symmetry decreases and finally at the $x=0.14$ (Mycielski et al. [67]) the inverted band structure changes into a direct one. The electronic properties of a narrow gap $\mathrm{Hg}_{1-v} \mathrm{Cd}_{v}$ Se crystals $(x \leq 0.25)$ are relatively well known (cf. Iwanowski et al. [68] and references therein). Optical and magnetooptical measurements of this material were performed by Summers and Broerman [69] and Mycielski et al. [67].

Mixed crystals of $\mathrm{Hg}_{1-v} \mathrm{Cd}_{v} \mathrm{Se}$ doped with $\mathrm{Fe}$ were for the first time investigated by Mycielski et al. [9]. Optical experiments including absorption and photo-emission studies, together with electron transport data for $\mathrm{HgSe}: \mathrm{Fe}$, showed that the energy of $\mathrm{Fe}^{2+}$ state depends on Cd content $x . \mathrm{Fe}^{2+}$ state is degenerate with the conduction band up to $x=0.4$ and for $x>0.4$ enters the energy gap.

The tuning of the location of $\mathrm{Fe}^{2+}$ level was applied by Wolff et al. [54] in four wave mixing experiment. The authors showed that an abrupt change of the scattering rate with an energy may be a reason for a third-order optical nonlinearity. Such sharp scattering changes are due to the presence of resonant donors in the conduction band. The experiment on $\mathrm{Hg}_{1-v} \mathrm{Cd}_{v} \mathrm{Se}: \mathrm{Fe}(x \approx 0.23)$ sample with the resonant $\mathrm{Fe}$ level located above the bottom of the conduction band revealed 
a large, free carrier induced, third-order optical nonlinearity. The magnitude of the effect was 20 times larger than the one that could have come from the second possible source of such nonlinearities - the nonparabolicity. On the basis of fitting of the calculated value of the free carrier absorption coefficient to the measured one the Fe level broadening was estimated to be $\Delta=20 \mathrm{meV}$ in $\mathrm{HgSe}: \mathrm{Fe}$ and $\Delta=$ $12 \mathrm{meV}$ in $\mathrm{Hg}_{1-v} \mathrm{Cd}_{v} \mathrm{Se}: \mathrm{Fe}$ for $x=0.23$.

$$
\text { 3.3. } I g_{1-x} Z n_{x} S e: F e
$$

In last years mixed crystals containing $\mathrm{Zn}$ gained an attention as a prospectus material for fabrication of infrared detectors (cf. Cobb et al. [70]). It is related to the fact that the presence of $\mathrm{Zn}$ improves mechanical properties of mercury-based II-VI semiconductors. A theoretical analysis of the lattice stability by Sher et al. [71] showed that weak HgTe (or HgSe) bonds are destabilized by an addition of $\mathrm{CdTe}$, but are made stronger by an addition of $\mathrm{ZnTe}$. The difference brought in by $\mathrm{Zn}$ and $\mathrm{Cd}$ comes from the fact that there is a net electron transfer from Cd to $\mathrm{Hg}$, while from $\mathrm{Zn}$ to $\mathrm{Hg}$ such transfer practically does not occur. Consequently, the vacancy formation energies in compounds containing $Z n$ should be larger than those in pure $\mathrm{HgTe}$ (or $\mathrm{HgSe}$ ). Cobb et al. [70] studied transport and optical properties of $\mathrm{Hg}_{1-x} \mathrm{Zn}_{x} \mathrm{Se}$ with $x<0.12$. Their conclusions confirm predictions of Sher and co-workers [71].

Galvanomagnetic and optical properties of $\operatorname{Ig}_{1-x} \mathrm{Zn}_{x}$ Se mixed crystals are well known (Cobb et al. [70] and references therein). At liquid helium temperature the triple degeneracy point of $\Gamma_{8}$ and $\Gamma_{6}$ bands was determined by Potapov et al. [72] to occur. at $\mathrm{Zn}$ content. The momentum matrix element $P$ was found to be dependent on the crystal composition, it changes rather rapidly with increasing $x$ from $7.1 \times 10^{-8} \mathrm{eV} \mathrm{cm}$ for IgSe to $7.9 \times 10^{-8} \mathrm{eV} \mathrm{cm}$ for $x=0.04$.

Measurements of the Hall effect and the conductivity were performed at temperatures ranging from 4.2 to $300 \mathrm{~K}$ on $\operatorname{Igg}_{1-x} \mathrm{Zn}_{x}$ Se samples with $x \leq 0.072$. The degree of doping with iron ranged from $n_{\mathrm{Fe}}=0$ to $n_{\mathrm{Fe}}=5 \times 10^{19} \mathrm{~cm}^{-3}$. Similarly to the observation in $\mathrm{HgSe}: \mathrm{Fe}$ or $\operatorname{IIg}_{1-x} \mathrm{Mn}_{x} \mathrm{Se}: \mathrm{Fe}$ an increase in the electron concentration with an increasing amount of $\mathrm{Fe}$ is observed, followed by a saturation at a sufficiently strong doping (Fig. 13).

The value of $n$ at the saturation depends on the $\mathrm{Zn}$ molar fraction. This results from a relative shift of the $\mathrm{Fe}$ resonant state energy and the bottom of the conduction band. Depending on the level of doping, an increase in the electron concentration (for heavily doped samples), a decrease (for lightly doped samples) or independence of the temperature is observed. It may be also noted that the temperature dependence of the electron concentration qualitatively resembles that observed in $\mathrm{HgSe}: \mathrm{Fe}$. The mobility values, particularly in the samples with a high concentration of $\mathrm{Fe}$, are relatively high. It is apparent that the observed values of the mobility (see Fig. 14) in the saturation region - corresponding to the mixed valence regime - are enhanced owing to the mechanism similar to that in $\mathrm{HgSe}: \mathrm{Fe}$.

The analysis of the mobility at liquid helium temperature was performed on an assumption that the two scattering mechanisms: the scattering from the ionized impurities and the alloy (chemical disorder) scattering are essential. The scattering by magnetic moments of Fe impurities (spin disorder scattering) was 


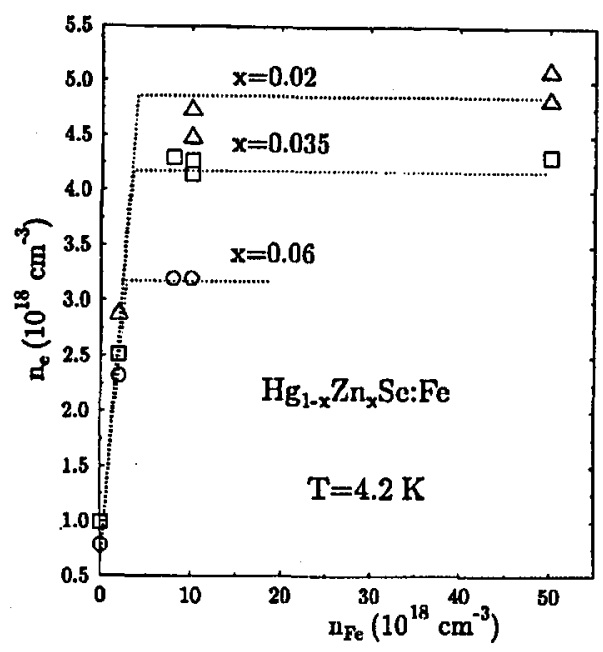

Fig. 13. The dependence of the electron concentration in $\mathrm{Hg}_{1-x} \mathrm{Zn}_{x}$ Se:Fe on the iron doping level for several sets of samples with different $\mathrm{Zn}$ contents. The lines are to guide the eye (after Ref. [56]).

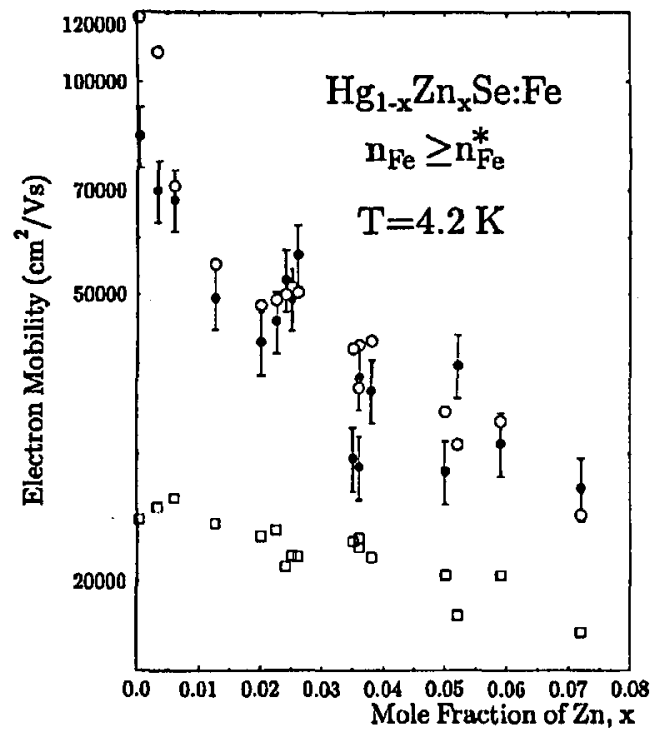

Fig. 14. The electron mobility in $\mathrm{Hg}_{1-x} \mathrm{Zn}_{x}$ Se at $4.2 \mathrm{~K}$ as a function of molar fraction $x$ for samples strongly doped with $\mathrm{Fe}\left(n_{\mathrm{Fe}}>n_{\mathrm{Fe}}^{*}\right)$. Experimental values are shown by full circles, values calculated taking into account screened ionized impurity scattering $\left(n_{\text {scatt }}=n_{\mathrm{e}}\right)$ and disorder scattering $(W=-0.5 \mathrm{eV}, V=2.6 \mathrm{eV})$ are shown by squares when no spatial correlation of impurities charges was included and by empty circles when the spatial correlation was accounted for within the short-range correlation model (after Ref. [56]). 
neglected, because this mechanism is weak (cf. previous sections), even in semimagnetic semiconductors in which concentration of magnetic moments exceeds by an order of magnitude the concentration of $\mathrm{Fe}$ in presently investigated samples. The calculated values of the mobility are shown by squares in Fig. 14. It is evident that in the case of the high doping corresponding to the mixed valence regime there is an efficient suppressing of the scattering rate, which we, again, relate to the spatial correlation of the impurity charges. When this mechanism was taken into account within the short-range correlation model the mobility values shown in Fig. 14 by circles were obtained. The agreement between the theory and the experiment is fair. This indicates that no other scattering mechanisms apart from already mentioned contribute significantly to the total scattering rate.

The mobility values found in the intentionally undoped samples of $\mathrm{Hg}_{1-x} \mathrm{Zn}_{x}$ Se are considerably smaller than those theoretically predicted. Obviously other scattering mechanisms impede the mobility. This fact additionally confirms that the presence of iron improves the electrical properties of HgSe based compounds, probably by making the formation of other defects less likely. The

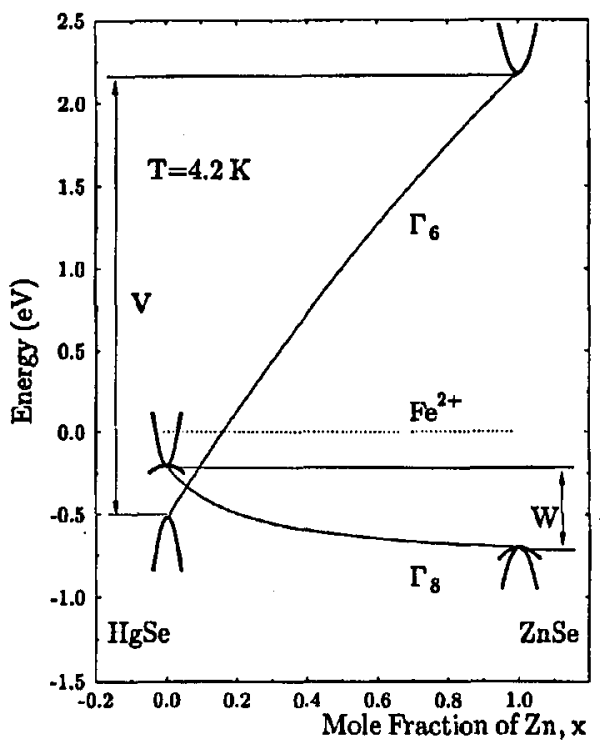

Fig. 15. Schematic representation of the conduction and valence band edges in $\mathrm{Hg}_{1-x} \mathrm{Zn}_{x} \mathrm{Se}$ as functions of the crystal composition. Dotted line shows the position of the Fe-related impurity level (independent of the composition according to Ref. [8]). Solid lines present the shift of $\Gamma_{6}$ and $\Gamma_{8}$ band edges upon the $\mathrm{Zn}$ content. The arrows marked by $V$ and $W$ show total energy difference between the positions of the $\Gamma_{6}$ and $\Gamma_{8}$ band edges, respectively, in the end point compounds. The energy of Fe level in $\mathrm{HgSe}$ and $\mathrm{ZnSe}$ taken from Ref. [9] and Refs. [73, 74] respectively (after Ref. [56]). 
improvement of electrical properties of the material is particularly clear when we compare the mobility values in the samples with no $\mathrm{Fe}$ dopant present with those in the samples having only a small amount of Fe. The mobility of the latter is substantially greater, closer to the value predicted theoretically by standard Brooks-Herring formula. Let us stress that in the samples doped only very slightly with $\mathrm{Fe}$ the Coulomb interaction driven correlation of charged impurity positions cannot arise, because all $\mathrm{Fe}$ donors are ionized.

Summarizing, the good quality of $\mathrm{Hg}_{1-x} \mathrm{Zn}_{x}$ Se crystals doped with Fe resonant donor can be obtained. Due to Fe doping the overall quality of the material improves. In particular, the electron mobility achieves high values (greater than those in samples doped with other impurities producing donors). This makes $\mathrm{Hg}_{1-x} \mathrm{Zn}_{x}$ Se particularly attractive for future applications.

Taking into account the relation between the energy gap and $\mathrm{Zn}$ mole fraction the Fermi energy for the samples in the saturation region (i.e., in the mixed valence regime) was calculated. Assuming that the Fermi level in these samples is pinned to the iron related resonant state and applying the Langer and Heinrich [8] rule, together with known (from the independent studies) energies of the $\mathrm{Fe}$ state in both end-compounds: $\mathrm{HgSe}$ and $\mathrm{ZnSe}\left(E_{\mathrm{Fe}}=0.2 \mathrm{eV}\right.$ above the bottom of the conduction band in $\mathrm{HgSe}$ [9]) and $E_{\mathrm{Fe}} \approx 0.6 \div 0.8 \mathrm{eV}$ above the top of the valence band in ZnSe (Szadkowski et al. [73] and Lubomirska-Wittlin et al. [74]), the shift of $\Gamma_{6}$ and $\Gamma_{8}$ band edges upon alloying with $\mathrm{Zn}$ was deduced. Figure 15 schematically shows this dependence. The difference between the values of $E_{\mathrm{Fe}}$ in $\mathrm{HgSe}$ and $\mathrm{ZnSe}$ with respect to the $\Gamma_{\mathbf{8}}$ band edges determines the parameter $W=$ $E_{\mathrm{Fe}}(\mathrm{HgSe})-E_{\mathrm{Fe}}(\mathrm{ZnSe}) \approx-0.4 \div-0.6 \mathrm{eV}$. The analogous difference with respect to the $\Gamma_{6}$ band edges determines the parameter $V=E_{\mathrm{Fe}}(\mathrm{ZnSe})-E_{\mathrm{Fe}}(\mathrm{HgSe}) \approx 2.6 \mathrm{eV}$.

$$
\text { 3.4. } H g S e_{1-x} T e_{x}: F e \text { and } H g S e_{1-x} S_{x}: F e
$$

The mixed crystals described in previous sections may be classified as alloys with mixed cations. The $\mathrm{Ig}$ ion in the crystal lattice is substituted by $\mathrm{Zn}$ or $\mathrm{Mn}$. The nearest neighborhood of $\mathrm{Fe}$ is unchanged and consists of four Se ions forming a perfect tetrahedron. Another situation will emerge when a mixed compound will contain $\mathrm{HgTe}$ or $\beta$ - $\mathrm{HgS}$ as a second component. In such alloy the nearest neighborhood of $\mathrm{Fe}$ atoms is modified and its symmetry is lowered. This may lead to a change of magnetic properties and to the so-called alloy splitting of the transition metal impurity state.

$\mathrm{HgTe}$ and $\beta$-HgS are known to have the band ordering of "inverted" type, the same as HgSe. While HgTe is undoubtfully the best known II-VI narrow gap semiconductor, $\beta$ - $\mathrm{HgS}$, on account of the fact that the zinc-blende phase of this semiconductor is unstable, is practically unknown. Both $\mathrm{HgTe}$ and $\mathrm{HgS}$ form solid solutions with $\mathrm{HgSe}$ in an arbitrary composition (Giriat [64]).

The experiments on the system $\mathrm{HgTe}-\mathrm{HgSe}$ doped with Fe were reported in by Dobrowolski et al. [26]. The Shubnikov-de Haas effect, low field Hall effect and resistivity were investigated on iron doped $\mathrm{HgSe}_{1-x} \mathrm{Te}_{x}$ samples $\left(n_{\mathrm{Fe}}<\right.$ $5 \times 10^{19} \mathrm{~cm}^{-3}, 0 \leq y \leq 0.53$ ) in wide temperature range.

The dependence of the effective mass on the electron concentration $n_{\mathrm{e}}$, derived from the Shubnikov-de Haas experiments, leads to a conclusion that the 
shape of the conduction band of $\mathrm{IIgSe}_{1-x} \mathrm{Te}_{x}$ does not differ noticeably from that of $\mathrm{HgSe}$. Thus it was assumed that the band parameters of $\mathrm{HgSe}$ described also $\mathrm{HgSe}_{1-x} \mathrm{Te}_{x}$ in the investigated composition range.

Figure 16 shows the electron concentration vs. Fe concentration for samples with $y=0.04, y=0.05$ and for HgSe (i.e. $y=0$ ).

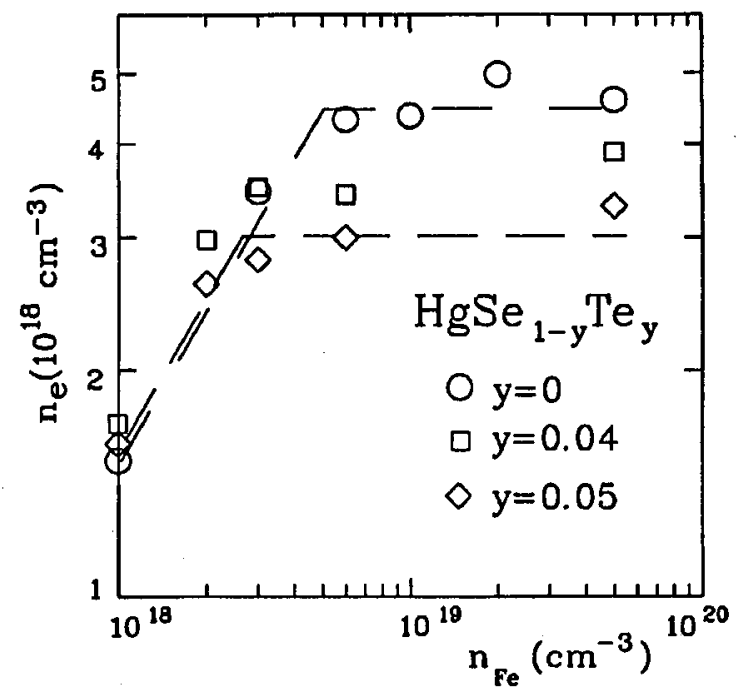

Fig. 16. Electron concentration in $\mathrm{HgSe}_{1-x} \mathrm{Te}_{x}$ as a function of Fe concentration at $4.2 \mathrm{~K}$ (after Ref. [26]).

The electron concentration at first increases with growing $n_{\mathrm{Fe}}$ to saturate when $n_{\mathrm{Fe}}$ exceeds a certain value depending on the molar fraction $y$. For $0.05<y<0.3$ all a vailable samples were already in the saturation region. The saturation level (which is due to the pinning of the Fermi level by the Fe state) varies with $y$. Its actual value enables us to determine the position of the Fe level as a function of Te concentration

$$
E_{\mathrm{Fe}}(y)=220-600 y[\mathrm{meV}] \text {. }
$$

The temperature dependence of the electron concentration in $y=0.04$ samples is shown in Fig. 17. In the mixed valence regime, i.e., for $n_{\mathrm{Fe}}>3 \times 10^{18} \mathrm{~cm}^{-3}$ we observe an increase in the concentration with an increase in temperature while samples with fewer Fe impurities present the decreasing or nearly constant concentration. This behavior is qualitatively similar to that observed in $\mathrm{HgSe}: \mathrm{Fe}$.

In the mixed valence regime the mobility is clearly enhanced compared to its values for low $n_{\mathrm{Fe}}$. In the crystals with Te the reduction of the charged donors scattering rate is by a factor 1.5-2 and appears to be somewhat weaker than in $\mathrm{HgSe}: \mathrm{Fe}$ (factor 3-4). Lower values of the mobility in samples with $y \neq 0$ indicate that some other scattering mechanism (apart from that by charged donors) is operative. Also a relatively strong variation of the mobility at liquid helium temperatures region evidences that, similarly to $\mathrm{IgSe}: \mathrm{Fe}$, the scattering by charged 


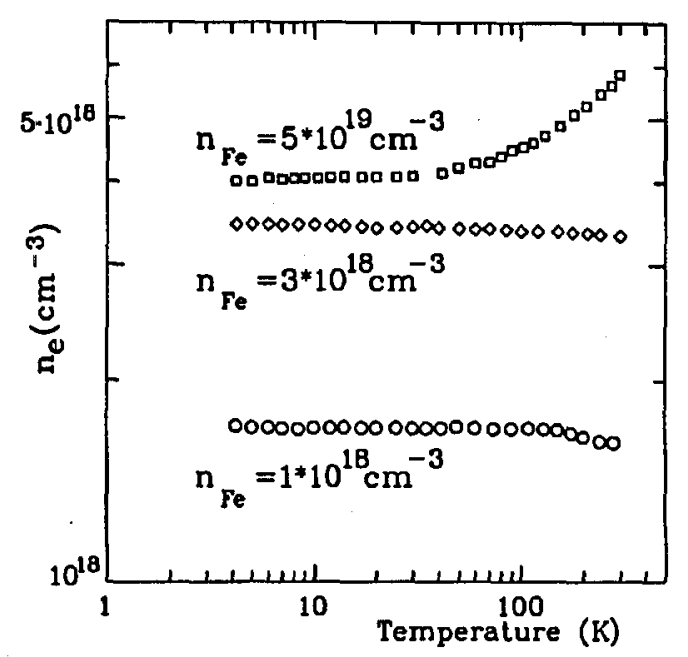

Fig. 17. Electron concentration in $\mathrm{HgSe}_{1-x} \mathrm{Te}_{x}(y=0.04)$ doped with $\mathrm{Fe}$ for various $\mathrm{Fe}$ contents. Circles: $n_{\mathrm{Fe}}=1 \times 10^{18} \mathrm{~cm}^{-3}$, asterisks: $n_{\mathrm{Fe}}=2 \times 10^{18} \mathrm{~cm}^{-3}$, diamonds: $n_{\mathrm{Fe}}=$ $3 \times 10^{18} \mathrm{~cm}^{-3}$, empty squares: $n_{\mathrm{Fe}}=6 \times 10^{18} \mathrm{~cm}^{-3}$, solid squares: $n_{\mathrm{Fe}}=5 \times 10^{19} \mathrm{~cm}^{-3}$. Lines are calculated assuming simple delta-like density of Fe states (after Ref. [26]).

donors is suppressed. The Dingle temperature (see Fig. 18), deduced from the analysis of the Shubnikov-de Haas oscillations, exhibits sudden drop when the mixed valence regime is reached, which additionally confirms that the scattering rate was reduced.

The position of the Fe level determined in the alloy system makes possible to estimate the valence and/or conduction band offsets applying the idea of Langer and Heinrich [8]. The resulting valence band offset in IIgTe-HgSe is $0.6 \mathrm{eV}$.

According to the trends present in the diagram of Langer and Heinrich [8] $\mathrm{Fe}^{2+}$ state is expected to have in cubic HgS energy higher than in the case of HgSe. The investigation of mixed crystals $\mathrm{HIgSe}_{1-x} \mathrm{~S}_{x}$ performed by Dybko [59] confirmed this prediction. The electron concentration observed at a very high Fe doping level (sufficient to pin the Fermi level to Fe state) increases with increasing $x$. Nevertheless, the study of the effects introduced by correlation are obstructed because the electron mobility decreases with increasing $x$, like in other mixed compounds, because of the alloy scattering. The temperature dependence of concentration in some samples shows nonmonotonic features, which can be attributed to the alloy splitting of the Fe state.

As already mentioned, any study of the end-compound $\beta$-HgS doped with $\mathrm{Fe}$, in which the effects connected with correlations should be observable, were impossible because the zinc-blende phase of $\mathrm{HgS}$ is metastable. Recently the situation has changed. Crystals of $\mathrm{Ig}_{1-x} \mathrm{Fe}_{x} \mathrm{~S}$ with $\mathrm{Fe}$ content $\dot{x}>0.01$ were successfully grown (Szuszkiewicz et al. [57]). The authors took advantage of the fact that the transition metal impurities have stabilizing effects on crystals of II-VI compounds (cf. Ref. [71]). The electron concentration in a sample with $x=0.01$ was found to be $n_{\mathrm{e}}=6.3 \times 10^{18} \mathrm{~cm}^{-3}$ at $4.2 \mathrm{~K}$. The corresponding electron mobility achieved 


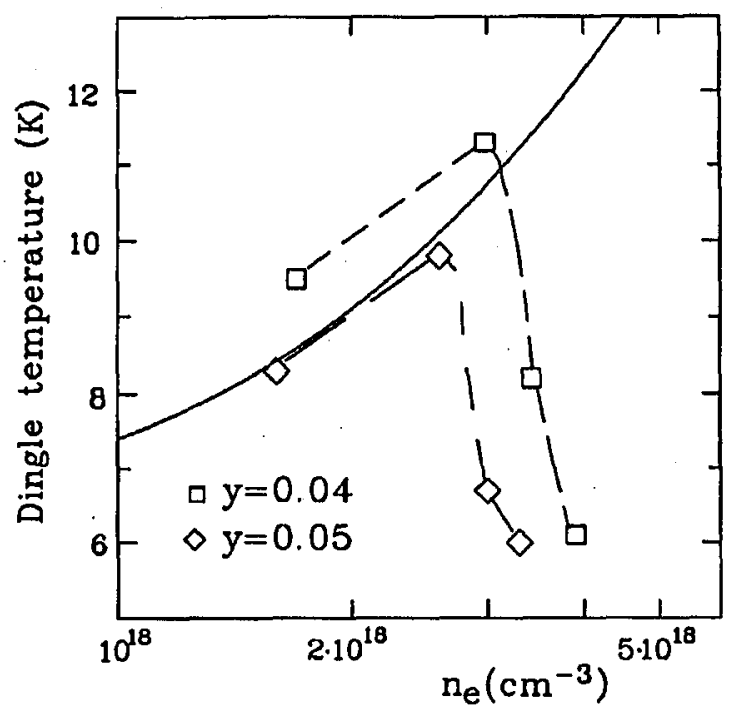

Fig. 18. The Dingle temperature in $\mathrm{HgSe}_{1-x} \mathrm{Te}_{x}$ (squares $y=0.04$ and diamonds $y=0.05$ ) from Shubnikov-de Haas amplitude in samples with various Fe contents as a function of the electron concentration. Solid line is calculated assuming scattering by random ionized donors (after Ref. [26]).

$16800 \mathrm{~cm}^{2} /(\mathrm{V} \mathrm{s})$ and the Dingle temperature $6.8 \pm 1.5 \mathrm{~K}$. Although the properties of $\beta$-HgS are poorly known and it is impossible to compare quantitatively scattering rate values in $\mathrm{HgS}$ and $\mathrm{Hg}_{1-x} \mathrm{Fe}_{x} \mathrm{~S}$ nevertheless the low value of the Dingle temperature suggests the existence of correlation effects also in $\mathrm{Hg}_{1-x} \mathrm{Fe}_{x} \mathrm{~S}$.

\section{Concluding remarks}

$\mathrm{HgSe}$ and HgSe based mixed crystals doped with $\mathrm{Fe}$ are unique systems. Due to the position of the $\mathrm{Fe}$ donor state in the conduction band low enough to be only partly filled up with electrons and appropriate other material parameters, the ionized $\mathrm{Fe}$ donors easily form spatially correlated systems. The experiments show that the mobility, as well as the Dingle temperature determined from the Shubnikov-de Haas oscillations, exhibit unusual values (respectively, "too" high and "too" low compared to an expectation based on standard theories) when only a fraction of $\mathrm{Fe}$ donors are in an ionized state. This fact is fully compatible with the hypothesis of the spatial correlation of electric charges localized on donors in the fractional occupation range of impurity concentrations. IIgSe based mixed crystals doped with $\mathrm{Fe}$ are at least qualitatively analogous to the parental binary $\mathrm{HgSe}$ doped with iron. There are, however, quantitative differences between $\mathrm{HgSe}: \mathrm{Fe}$ and its alloys with other semiconductors. The reduction of the scattering rate at low temperatures is by far more striking in the $\mathrm{HgSe}: \mathrm{Fe}$ compound. These differences are related to the presence of other important scattering mechanisms that retard the motion of charge carriers in ternary alloys: the chemical disorder scattering and (in the case of Mn-containing compounds) spin disorder scattering. 
The investigation of $\mathrm{HgSe}: \mathrm{Fe}$ were recently extended on samples grown in non-equilibrium processes. Widmer et al. [75] reported a successful epitaxial growth of Fe doped IIgSe layers and microstructures in a specially designed MBE machine. The preliminary electron transport data obtained from Hall effect and Shubnikov-de Haas measurements indicate to a very high quality of the samples. The highest measured mobility value $\left(2.7 \times 10^{6} \mathrm{~cm}^{2} /(\mathrm{V} \mathrm{s})\right)$ is almost two times higher than that known from the studies of bulk samples. A very interesting feature was observed in an angular dependence of the Shubnikov-de Haas amplitude. The change of the orientation of the magnetic field from the normal to the parallel one weakened the amplitude of oscllations in a $\mathrm{HgSe}: \mathrm{Fe}$ layer and caused that the amplitude vanished completely in a microstructure (alternating $580 \AA \mathrm{HgSe}$ and $730 \AA \mathrm{HgSe}: \mathrm{Fe})$. The authors suggest that this effect is due to a higher scattering rate when the cyclotron orbit enters the lower mobility $\mathrm{HgSe}$ region. Such behavior, according to the authors, indicates that the von Ortenberg [76] idea of scattering superlattices was realized.

In $\mathrm{HgSe}$ based alloys doped with $\mathrm{Fe}$ one may expect an appearance of the effect known as the alloy splitting. This effect arises when the impurities can be found in various environments, i.e. with a different chemical composition in surrounding shells of atoms. Such splitting was previously clearly observed e.g. in $\mathrm{Ga}_{1-x} \mathrm{Al}_{x} \mathrm{As}$ doped with Si by Wilamowski et al. [34] and Ostermayer et al. [77]. The alloy splitting is responsible for a smaller degree of the scattering rate reduction due to the spatial correlation of impurity charges. The absence of this effect in cases when an impurity in the host lattice substitutes a cation which constitutes the second neighboring shell surrounding an $\mathrm{Fe}$ ion is not surprising at all. The marks of the alloy splitting were seen by Dybko [59] in the two anion alloy $\mathrm{HgSe}_{1-x} \mathrm{~S}_{x}: \mathrm{Fe}$. The complex temperature dependence of the electron concentration observed in $\mathrm{HgSe}_{1-x} \mathrm{~S}_{x}: \mathrm{Fe}$ is difficult to understand without assuming an existence of a few additional density of states pockets superimposed on the conduction band continuum. In this context the lack of evidence of alloy splitting in other compound with mixed anions $\mathrm{HgSe}_{1-x} \mathrm{Te}_{x}$ :Fe may be ascribed to an unfavorable condition to observe this effect, for instance a small value of splitting.

Finally, it may be noted that eflects similar to those observed in $\mathrm{HgSe}: \mathrm{Fe}$ should be in principle expected in other semiconductors. IV-VI semiconducting compounds, where many impurities act as donors resonant with the conduction hand (cf. Akimov et al. [78]), seem to be good candidates. The dependencies of electron concentrations on levels of doping or temperature conduct themselves in the same way as in $\mathrm{HgSe}: \mathrm{Fe}$. Nevertheless, on account of the very high dielectric constant in these materials (almost two orders of magnitude higher than in $\mathrm{HgSe}$ ) the Coulomb interaction between ions is weak. An estimate of a characteristic temperature of an appearance of correlations within the short-range correlation model gives in $\mathrm{PbTe}: \mathrm{Cr} T^{*}=E_{\mathrm{c}} / k_{\mathrm{B}}=3.5 \mathrm{~K}$, where $E_{\mathrm{c}}$ is the energy gain (per charged impurity) due to the spatial correlation of impurity charges. For comparison, the value of $T^{*}$ in $\mathrm{HgSe}: \mathrm{Fe}$ is about $70 \mathrm{~K}$ (Grodzicka et al. [79]). In $\mathrm{PbTe}: \mathrm{Cr}$ there was no unambiguously any characteristic feature of the enhanced mobility (e.g., strong temperature variation of the mobility in the region in which the correlation forms) down to the lowest temperatures studied. 
In the classical narrow gap semiconducting compound, InSb, in which at least three impurities form resonant donors, namely, S, Se and Te (cf. Kończewicz et al. $[80,81])$ another factor makes the existence of the correlation impossible. The donor states are located too high in the conduction band to be adequately filled up with electrons.

It should be mentioned that also in compensated semiconductors exist conditions providing the occurrence of spatial correlations of impurities (cf. Reiss et al. [82] and Stratton [83]). Good examples are the zero gap $\mathrm{Hg}_{1-x} \mathrm{Mn}_{x} \mathrm{Te}$ and $\mathrm{IIg}_{1-x} \mathrm{Cd}_{x} \mathrm{Te}$ mixed crystals. When the Fermi level is pinned to resonant acceptors (traps) ionized donors are likely to be in a neighborhood of an ionized acceptor because of mutual Coulomb attraction. Thus a system of dipoles develops which scatters electrons less effectively than ionized donors do (Arapov et al. [84] and Brandt et al. [85]). Another system, in which a dipole arrangement provides an explanation for the mobility increase, is $\mathrm{Al}_{1-x} \mathrm{Ga}_{x} \mathrm{As}$ with DX centers (Wilamowski et al. [86] and references therein).

Thinking about perspective of further investigations of $\mathrm{HgSe}: \mathrm{Fe}$ and related compounds at least two directions may be named. With the first successful growth of $\mathrm{HgSe}: \mathrm{Fe}$ layers using MBE method the new fields of investigations were established. Undoubtfully, it opens many fascinating research opportunities. But there exists also another issue which needs to be explored and explained. The recent result of Dybko et al. [52] showed that under certain conditions magnetic properties of $\mathrm{HgSe}: \mathrm{Fe}$ are not the simple sum of the contributions given by the magnetic subsystem composed of the $\mathrm{Fe}^{2+}$ and the $\mathrm{Fe}^{3+}$ ions. This experimental finding suggests that there exists an interaction of those two subsystems. It is worth noting that both subsystems are strongly diluted, and each of them may be regarded as one composed of noninteracting spins. To solve the puzzle what is the nature of the magnetic interaction between subsystems, experimental and theoretical efforts should be undertaken. Investigation of magnetic properties of mixed crystals, such as $\mathrm{Hg}_{1-x} \mathrm{Mn}_{x}$ Se doped with $\mathrm{Fe}$ seems to be here of a great help.

Investigation of HgSe:Fe and related mixed crystals brought a number of new experimental and theoretical facts connected with resonant states, mixed valency or spatial correlations in semiconductors. Many of these results were of broader meaning in semiconductor physics and were applied to the more general problems of doping and behavior of impurity states in semiconductors, significantly extending the knowledge of the properties of heavy doped materials.

\section{Acknowledgments}

I gratefully acknowledge fruitful cooperation and helpful discussions with K. Buchholz-Stepputtis, T. Dietl, K. Dybko, R.R. Gałązka, E. Grodzicka, J. Kossut, T. Lenard, A. Mycielski, M. von Ortenberg, O. Portugall, A. Reich, C. Skierbiszewski, Z. Wilamowski and B. Witkowska.

\section{References}

[1] R.R. Gałazka, W.M. Becker, D.G. Seiler, J. Phys. Chem. Solids 32, 481 (1971).

[2] D.G. Seiler, R.R. Gałazka, W.M. Becker, Phys. Rev. B 3, 4274 (1971). 
[3] S.H. Groves, W. Paul, Phys. Rev. Lett. 11, 194 (1963).

[4] J. Kossut, W. Dobrowolski, in: Handbook of Magnetic Materials, Vol. 7, Ed. K.H.J. Buschow, Elsevier, Amsterdam 1993, Ch. 4, p. 231.

[5] T. Dietl, in: Handbook on Semiconductors, Eds. T.S. Moss, S. Mahajan, Vol. 3, Elsevier, Amsterdam 1994, Ch. 17, p. 1251.

[6] A. Mycielski, J. Appl. Phys. 63, 3279 (1988).

[7] S.M. Komirenko, Y.G. Semenov, A.E. Belyaev, Y.S. Ryabchenko, Phys. Rev. B 50, 14131 (1994).

[8] J.M. Langer, H. Heinrich, Phys. Rev. Lett. 55, 1414 (1985).

[9] A. Mycielski, P. Dzwonkowski, B. Kowalski, B.A. Orłowski, M. Dobrowolska, M. Arciszewska, W. Dobrowolski, J.M. Baranowski, J. Phys. C, Solid State Phys. 19, 3605 (1986).

[10] F.S. Pool, J. Kossut, U. Debska, R. Reifenberger, Phys. Rev. B 35, 3900 (1987).

[11] W. Dobrowolski, K. Dybko, A. Mycielski, J. Mycielski, J. Wróbel, S. Piechota, M. Palczewska, H. Szymczak, Z. Wilamowski, in: Proc. 18th Int. Conf. on Phys. Semicond., Stockholm 1986, Ed. O. Engström, Vol. 2, World Scientific, Singapore 1987, p. 1743.

[12] A. Reich, W. Dobrowolski, J. Raułuszkiewicz, Acta Phys. Pol. A 73, 479 (1988).

[13] B.J. Kowalski, V. Chab, B.A. Orłowski, J. Majewski, A. Sarem, A. Mycielski, Acta Phys. Pol. A 73, 455 (1988).

[14] W. Szuszkiewicz, Q. Dingrong, C. Julien, Z. Jiaming, M. Jezewski, M. Baikanski, A. Mycielski, J. Cryst. Growth 101, 507 (1990).

[15] M. v. Ortenberg, O. Portugall, W. Dobrowolski, A. Mycielski, R.R. Gałązka, F. Herlach, J. Phys. C, Solid State Phys. 21, 5393 (1988).

[16] J. Kossut, W. Dobrowolski, Acta Phys. Pol. A 75, 261 (1989).

[17] Z. Wilamowski, A. Mycielski, W. Jantsch, G. Hendorfer, in: 19 Int. Conf. on Phys. of Semicond., Warsaw 1988, Ed. W. Zawadzki, Vol. 2, Inst. of Phys., Pol. Acad of Sciences, Warsaw 1988, p. 1225.

[18] I. Nowik, E.R. Bauminger, A. Mycielski, H. Szymczak, Physica B 153, 215 (1988).

[19] A. Mycielski, W. Dobrowolski, J. Wróbel, unpublished.

[20] M. Vaziri, R. Reifenberger, Phys. Rev. B 32, 3921 (1985).

[21] M.M. Miller, R. Reifenberger, Phys. Rev. B 38, 3423 (1988).

[22] M.M. Miller, R. Reifenberger, Phys. Rev. B 38, 4120 (1988).

[23] O. Portugall, H. Yokoi, S. Takeyama, L. v. Bockstal, K. Buchholz-Stepputtis, M. v. Ortenberg, F. Herlach, W. Dobrowolski, in: Proc. 20th Int. Conf. on Phys. Semicond. Thessaloniki 1990, Eds. E.M. Anastassakis, J.D. Joannopulos, World Scientific, Singapore 1990, p. 2287.

[24] K. Buchholz-Stepputtis, O. Portugall, M. v. Ortenberg, W. Dobrowolski, in: Conference Digest: Sixteenth Int. Conf. on Infrared and Millimeter Waves, Eds. M.R. Siegrist, M.Q. Tran, T.M. Tran, Centre de Recherches en Physique des Plasmas - Ecole Polytechnique Fédérale de Lausanne, Lausanne 1991, p. 159.

[25] K. Buchholz-Stepputtis, O. Portugall, M. v. Ortenberg, W. Dobrowolski, in: Proc. 1th Int. Conf. on Phys. Semicond., Bejing 1992, Eds. P. Jiang, H.-Z. Zheng, World Scientific, Singapore 1992, p. 1844. 
[26] W. Dobrowolski, K. Dybko, C. Skierbiszewski, T. Suski, E. Litwin-Staszewska, S. Miotkowska, J. Kossut, A. Mycielski, in: 19 Int. Conf. on Phys. of Semicond., Warsaw 1988, Ed. W. Zawadzki, Vol. 2, Inst. of Phys., Pol. Acad. of Sciences, Warsaw 1988, p. 1247.

[27] C. Skierbiszewski, T. Suski, E. Litwin-Staszewska, W. Dobrowolski, K. Dybko, A. Mycielski, Semicond. Sci. Technol. 4, 293 (1989).

[28] T. Dietl, W. Szymańska, J. Phys. Chem. Solids 39, 1041 (1978).

[29] A. Lenard, M. Arciszewska, T. Dietl, M. Sawicki, W. Plesiewicz, T. Skośkiewicz, W. Dobrowolski, K. Dybko, Physica B 165-166, 219 (1990).

[30] A. Lenard, T. Dietl, M. Sawicki, W. Dobrowolski, K. Dybko, T. Skośkiewicz, W. Plesiewicz, S. Miotkowska, A. Witek, A. Mycielski, J. Low Temp. Phys. 80, 15 (1990).

[31] J. Mycielski, Solid State Commun. 60, 165 (1986).

[32] T. Dietl, Jpn. J. Appl. Phys. 26 Suppl., 26 (1987).

[33] A.L. Efros, B.I. Shklovskii, in: Electron-Electron Interaction in Disordered Systems, North-Holland, Amsterdam 1985, p. 409.

[34] Z. Wilamowski, K. Świątek, T. Dietl, J. Kossut, Solid State Commun. 74, 833 (1990).

[35] E.O. Kane, J. Phys. Chem. Solids 1, 249 (1957).

[36] I.M. Tsidil'kovski Y, I.G. Kuleev, I.I. Lyapilin, JETP 75, 172 (1992).

[37] J. Kossut, Phys. Status Solidi B 72, 359 (1975).

[38] J. Kossut, Phys. Status Solidi B 86, 593 (1978).

[39] C. Skierbiszewski, Z. Wilamowski, J.'Kossut, Semicond. Sci. Technol. 8, S22 (1993).

[40] I.G. Kuleev, A.T. Lonchakov, I.I. Lyapilin, I.M. Tsidil'kovski ̌, Zh. Eksp. Teor. Fiz. 103, 248 (1993).

[41] H. Serre, G. Bastard, C. Rigaux, J. Mycielski, J.K. Furdyna, in: Physics of Narrow Gap Semiconductors, Vol. 52 of Lecture Notes in Physics, Eds. E. Gornik, H. Heinrich, L. Palmetshofer, Springer-Verlag, Berlin 1982, p. 321.

[42] O. Portugall, J. Kono, Y. Shimamoto, N. Miura, R. Shoenfeld, M. v. Ortenberg, W. Dobrowolski, in: Proc. Yamada Conf. XXXIII on the Application of the High Magnetic Fields in Semiconductor Plysics, Chiba (Japan) 1992, Ed. N. Miura, Physica $B$ 184, 465 (1992).

[43] M. v. Ortenberg, Semicond. Sci. Technol. 8, S163 (1993).

[44] W. Szuszkiewicz, W. Bardyszewski, Q. Dingrong, Z. Jiaming, C. Julien, M. Balkanski, B. Witkowska, A. Mycielski, in: Proc. 20th Int. Conf. on Phys. Semicond., Thessaloniki 1990, Eds. E.M. Anastassakis, J.D. Joannopulos, World Scientific, Singapore 1990, p. 2255.

[45] W. Szuszkiewicz, P. Sobkowicz, B. Witkowska, W. Bardyszewski, C. Julien, M. Balkanski, Acta Phys. Pol. A 84, 559 (1993).

[46] A. Lewicki, J. Spałek, A. Mycielski, J. Phys. C, Solid State Phys. 20, 2005 (1987).

[47] M. Arciszewska, A. Lenard, T. Dietl, W. Plesiewicz, T. Skośkiewicz, W. Dobrowolski, Acta Phys. Pol. A 77, 155 (1990).

[48] M. Arciszewska, Acta Phys. Pol. A 70, 341 (1991).

[49] M. Arciszewska, Ph.D. Thesis, Institute of Physics, Polish Academy of Sciences, Warszawa 1993. 
[50] M. v. Ortenberg, O. Portugall, W. Dobrowolski, A. Mycielski, R.R. Gałazka, in: 19th Int. Conf. on Phys. of Semicond., Warsaw 1988, Ed. W. Zawadzki, Vol. 2, Inst. of Phys., Pol. Acad. of Sciences, Warsaw 1988, p. 1575.

[51] Z. Wilamowski, H. Przybylińska, W. Joss, M. Guillot, Semicond. Sci. Technol. 8, S44 (1993).

[52] K. Dybko, A. Lusakowski, J. Kossut, M. Arciszewska, P.J.E.M. v. d. Linden, J.A.A.J. Perenboom, A. Mycielski, Acta Phys. Pol. A 87, 193 (1995).

[53] U. Zeitler, S.A.J. Wiegers, M.R.C. Overkamp, A. Wittlin, J.C. Maan, W. Dobrowolski, A. Mycielski, Physica B 211, 381 (1995).

[54] P.A. Wolff, S.Y. Auyang, R.R. Galazka, A. Mycielski, J. Vac. Sci. Technol. A 6, 2696 (1988).

[55] W. Dobrowolski, E. Grodzicka, J. Kossut, B. Witkowska, Semicond. Sci. Technol. 8, S33 (1993).

[56] W. Dobrowolski, R.R. Galazka, E. Grodzicka, J. Kossut, B. Witkowska, Phys. Rev. $B$ 48, 17848 (1993).

[57] W. Szuszkiewicz, B. Witkowska, M. Jouanne, Acta Phys. Pol. A 87, 415 (1995).

[58] W. Szuszkiewicz, E. Guziewicz, B. Kowalski, B. Orłowski, B. Witkowska, Mater. Sci. Forum (Switzerland) 182-184, 707 (1995).

[59] K. Dybko, Ph.D. Thesis, Institute of Physics, Polish Academy of Sciences, Warszawa 1995.

[60] W. Dobrowolski, J. Kossut, B. Witkowska, R.R. Galązka, in: High Magnetic Fields in Semiconductor Physics II, Vol. 87 of Springer Series in Solid-State Science, Ed. G. Landwehr, Springer-Verlag, Berlin 1989, p. 496.

[61] M. v. Ortenberg, N. Miura, W. Dobrowolski, Semicond. Sci. Technol. 5, 274 (1990).

[62] W. Dobrowolski, J. Kossut, B. Witkowska, R. Gałazkka, Acta Phys. Pol. A 77, 151 (1990).

[63] E. Crucheanu, D. Niculescu, N. Nistor, Rev. Phys. Acad. Rep. Pop. Roumaine 8, 379 (1963).

[64] W. Giriat, Phys. Lett. A 26, 639 (1968).

[65] R.R. Gałazka, J. Kossut, in: Semiconductors, Vol.17b of Landolt-Börnstein, New Series, Group III, Ed. O. Madelung, Springer-Verlag, Berlin 1982, Ch. 5, p. 302.

[66] M. Dobrowolska, W. Dobrowolski, R.R. Gałazka, A. Mycielski, Phys. Status Solidi $B$ 105, 447 (1981).

[67] A. Mycielski, J. Kossut, M. Dobrowolska, W. Dobrowolski, J. Phys. C, Solid State Phys. 15, 3293 (1982).

[68] R. Iwanowski, T. Dietl, W. Szymaniska, J. Phys. Chem. Solids 39, 1059 (1978).

[69] C.J. Summers, J.G. Broerman, Phys. Rev. B 21, 559 (1980).

[70] S.D. Cobb, R.N. Andrews, F.R. Szofran, S.L. Lehoczky, J. Cryst. Growth 110, 415 (1991).

[71] A. Sher, A.-B. Chen, W.E. Spicer, C.-K. Shih, J. Vac. Sci. Technol. A 3, 105 (1985).

[72] G.A. Potapov, A.I. Ponomarev, N.P. Gaveleshko, V.V. Khomyak, Fiz. Tekh. Poluprovodn. 14, 2333 (1980).

[73] A.J. Szadkowski, A. Lubomirska-Wittlin, A. Zaręba, M. Demianiuk, Acta Phys. Pol. A 77, 163 (1990). 
[74] A. Lubomirska-Wittlin, A. Szadkowski, M. Demianiuk, Acta Phys. Pol, A 79, 337 (1991).

[75] T. Widmer, D. Schikora, G. Hendorfer, S. Luther, W. Jantsch, K. Lischka, M. v. Ortenberg, Mater. Sci. Forum 182-184, 395 (1995).

[76] M. v. Ortenberg, Adv. in Solid State Phys. 31, 261 (1990).

[77] G. Ostermayer, W. Jantsch, D. Dobosz, Z.R. Żytkiewicz, Z. Wilamowski, Acta Phys. Pol. A 84, 769 (1993).

[78] B.A. Akimov, A.V. Dmitriev, D.R. Khokhlov, L.I. Ryabova, Phys. Status Solidi A 137, 9 (1993).

[79] E. Grodzicka, W. Dobrowolski, J. Kossut, T. Story, B. Witkowska, J. Cryst. Growth 138, 1034 (1994).

[80] L. Konczewicz, E. Litwin-Staszewska, S. Porowski, in: Proc. III Int. Conf. on Phys. of Narrow Gap Semiconductors, Warszawa 1978, Eds. J. Raułuszkiewicz, M. Górska, E. Kaczmarek, PWN, Warszawa 1978, p. 211.

[81] L. Kończewicz, W. Trzeciakowski, Phys. Status Solidi B 115, 359 (1983).

[82] H. Reiss, C.S. Fuller, F.J. Morin, Bell. Syst. Tech. J. 35, 535 (1956).

[83] R. Stratton, J. Phys. Chem. Solids 23, 1011 (1962).

[84] Y.G. Arapov, F.I. Akhmedova, A.B. Davydov, I.M. Tsidil'kovskiY, Sov. Phys. Semicond. 16, 32 (1982).

[85] N.B. Brandt, V.V. Moshchalkov, A.O. Orlov, L. Skrbek, I.M. Tsidil'kovski Y, S.M. Chudinov, Sov. Phys.-JETP 57, 614 (1983).

[86] Z. Wilamowski, W. Jantsch, G. Ostermayer, J. Kossut, Mater. Sci. Forum 83-87, 805 (1992).

[87] G. Gluzman, L.D. Sabirzyanova, I.M. Tsidil'kovskiy, L.D. Paranchich, S.Y. Paranchich, Sov. Phys.-Semicond. 20, 1251 (1986).

[88] M. Dobrowolska, W. Dobrowolski, A. Mycielski, Solid State Commun. 34, 441 (1980). 